\title{
Herschel-PACS photometry of faint stars for sensitivity performance assessment and establishment of faint FIR primary photometric standards ${ }^{\star, \star \star}$
}

\author{
U. Klaas ${ }^{1}$, Z. Balog ${ }^{1}$, M. Nielbock ${ }^{1,2}$, T.G. Müller ${ }^{3}$, H. Linz ${ }^{1}$, and Cs. Kiss ${ }^{4}$ \\ ${ }^{1}$ Max-Planck-Institut für Astronomie (MPIA), Königstuhl 17, 69117 Heidelberg, Germany \\ e-mail: klaas@mpia.de \\ ${ }^{2}$ Haus der Astronomie, MPIA-Campus, Königstuhl 17, 69117 Heidelberg, Germany \\ ${ }^{3}$ Max-Planck-Institut für extraterrestrische Physik (MPE), PO Box 1312, Giessenbachstraße, 85741 Garching, Germany \\ ${ }^{4}$ Konkoly Observatory, Research Centre for Astronomy and Earth Sciences, Hungarian Academy of Sciences, \\ Konkoly Thege Miklós út 15-17, 1121 Budapest, Hungary
}

Received 22 August 2017 / Accepted 4 December 2017

\begin{abstract}
Aims. Our aims are to determine flux densities and their photometric accuracy for a set of seventeen stars that range in flux from intermediately bright $(\$ 2.5 \mathrm{Jy})$ to faint $(\gtrsim 5 \mathrm{mJy})$ in the far-infrared (FIR). We also aim to derive signal-to-noise dependence with flux and time, and compare the results with predictions from the Herschel exposure-time calculation tool.

Methods. We obtain aperture photometry from Herschel-PACS high-pass-filtered scan maps and chop/nod observations of the faint stars. The issues of detection limits and sky confusion noise are addressed by comparison of the field-of-view at different wavelengths, by multi-aperture photometry, by special processing of the maps to preserve extended emission, and with the help of large-scale absolute sky brightness maps from AKARI. This photometry is compared with flux-density predictions based on photospheric models for these stars. We obtain a robust noise estimate by fitting the flux distribution per map pixel histogram for the area around the stars, scaling it for the applied aperture size and correcting for noise correlation.

Results. For 15 stars we obtain reliable photometry in at least one PACS filter, and for 11 stars we achieve this in all three PACS filters $(70,100,160 \mu \mathrm{m})$. Faintest fluxes, for which the photometry still has good quality, are about 10-20 mJy with scan map photometry. The photometry of seven stars is consistent with models or flux predictions for pure photospheric emission, making them good primary standard candidates. Two stars exhibit source-intrinsic far-infrared excess: $\beta$ Gem (Pollux), being the host star of a confirmed Jupitersize exoplanet, due to emission of an associated dust disk, and $\eta$ Dra due to dust emission in a binary system with a K1 dwarf. The investigation of the $160 \mu \mathrm{m}$ sky background and environment of four sources reveals significant sky confusion prohibiting the determination of an accurate stellar flux at this wavelength. As a good model approximation, for nine stars we obtain scaling factors of the continuum flux models of four PACS fiducial standards with the same or quite similar spectral type. We can verify a linear dependence of signal-to-noise ratio $(\mathrm{S} / \mathrm{N})$ with flux and with square root of time over significant ranges. At $160 \mu \mathrm{m}$ the latter relation is, however, affected by confusion noise.

Conclusions. The PACS faint star sample has allowed a comprehensive sensitivity assessment of the PACS photometer. Accurate photometry allows us to establish a set of five FIR primary standard candidates, namely $\alpha$ Ari, $\varepsilon$ Lep, $\omega$ Cap, HD 41047 and 42 Dra, which are 2-20 times fainter than the faintest PACS fiducial standard ( $\gamma$ Dra) with absolute accuracy of $<6 \%$. For three of these primary standard candidates, essential stellar parameters are known, meaning that a dedicated flux model code may be run.
\end{abstract}

Key words. space vehicles: instruments - methods: data analysis - techniques: photometric - infrared: stars - stars: atmospheres radiation mechanisms: thermal

\section{Introduction}

The photometric calibration of the PACS photometer (Poglitsch et al. 2010) on-board the Herschel Space Observatory (Pilbratt et al. 2010) is based on celestial standard stars (Balog et al. 2014; Nielbock et al. 2013). These primary standard stars have well-modelled spectral energy distributions (SEDs) of their photospheric emission and an accurate absolute calibration in the $K$-band (Dehaes et al. 2011). They are still relatively bright in

\footnotetext{
${ }^{\star}$ Herschel is an ESA space observatory with science instruments provided by European-led Principal Investigator consortia and with important participation from NASA.

$\star \star$ Tables A.3 to A.5 and B.1 to B.3 are only available in electronic form at the CDS via anonymous ftp to cdsarc.u-strasbg. fr (130.79.128.5) or via

http://cdsarc.u-strasbg.fr/viz-bin/qcat?J/A+A/613/A40
}

the far-infrared (in the range 1-10 Jy) to achieve high signalto-noise ratios $(\mathrm{S} / \mathrm{Ns})$ within reasonable measurement times. Besides repeated measurements of these standard stars, a set of fainter secondary standard stars was repeatedly measured by PACS as part of the calibration program during the Herschel Performance Verification and Routine Operations periods. This included sources down to a few mJy. The PACS photometer is linear over a flux range exceeding the primary standard fluxes, with an optimized detector set-up for the flux background from the telescope. Flux nonlinearity is therefore an issue for considerably brighter sources and has been addressed elsewhere (Müller et al. 2016). However, including fainter sources with well known flux predictions allows to us address the following questions:

1) How does the sensitivity scale with flux and time? 
Table 1. Faint secondary standards observed by Herschel-PACS.

\begin{tabular}{|c|c|c|c|c|c|c|}
\hline \multirow[b]{2}{*}{ HD } & \multirow[b]{2}{*}{ Other name } & \multicolumn{3}{|c|}{ Model flux prediction (mJy) } & \multirow[t]{2}{*}{ Spectral type } & \multirow[t]{2}{*}{ Reference } \\
\hline & & $\mathrm{f}_{70}$ & $\mathrm{f}_{100}$ & $\mathrm{f}_{160}$ & & \\
\hline 62509 & $\beta \mathrm{Gem}$ & $2457( \pm 5.73 \%)$ & $1190( \pm 5.73 \%)$ & $455.9( \pm 5.73 \%)$ & K0IIIb & Cohen et al. (1996) ${ }^{a}$ \\
\hline 12929 & $\alpha$ Ari & $1707( \pm 5.9 \%)$ & $831.4( \pm 5.9 \%)$ & $321.0( \pm 5.9 \%)$ & K2III & Cohen et al. $(1996)^{a}$ \\
\hline 32887 & $\varepsilon$ Lep & $1182( \pm 5.9 \%)$ & $576.2( \pm 5.9 \%)$ & $222.7( \pm 5.9 \%)$ & K4III & Cohen et al. (1996) ${ }^{a}$ \\
\hline 198542 & $\omega$ Cap & $857.7( \pm 6.03 \%)$ & $418.0( \pm 6.03 \%)$ & $161.5( \pm 6.03 \%)$ & MOIII & Cohen et al. (1996) ${ }^{a}$ \\
\hline 148387 & $\eta$ Dra & $479.5( \pm 3.38 \%)$ & $232.6( \pm 3.45 \%)$ & $89.4( \pm 3.51 \%)$ & G8III & Hammersley et al. $(1998)^{b}$ \\
\hline 180711 & $\delta$ Dra & $428.9( \pm 5.7 \%)$ & $207.7( \pm 5.7 \%)$ & $79.6( \pm 5.7 \%)$ & G9III & Cohen et al. $(1996)^{a}$ \\
\hline 139669 & $\theta \mathrm{Umi}$ & $286.2( \pm 5.67 \%)$ & $139.5( \pm 5.67 \%)$ & $53.9( \pm 5.67 \%)$ & K5III & Cohen et al. (1996) ${ }^{a}$ \\
\hline 41047 & HR 2131 & $195.6( \pm 5.96 \%)$ & $95.4( \pm 5.96 \%)$ & $36.9( \pm 5.96 \%)$ & K5III & Cohen et al. (1996) ${ }^{a}$ \\
\hline 170693 & 42 Dra & $153.7 \pm 4.6$ & $75.3( \pm 3.0 \%)$ & $29.4( \pm 3.0 \%)$ & K1.5III & Gordon et al. (2007) \\
\hline 138265 & HR 5755 & $115.9 \pm 4.0$ & $56.8( \pm 3.5 \%)$ & $22.2( \pm 3.5 \%)$ & K5III & Gordon et al. (2007) \\
\hline 159330 & HR 6540 & $64.2 \pm 2.1$ & $31.5( \pm 3.3 \%)$ & $12.3( \pm 3.3 \%)$ & K2III & Gordon et al. (2007) \\
\hline 152222 & SAO 17226 & $39.4 \pm 1.9$ & $19.3( \pm 5.0 \%)$ & $7.5( \pm 5.0 \%)$ & K2III & Gordon et al. (2007) \\
\hline 39608 & SAO 249364 & $30.9 \pm 1.2$ & $15.1( \pm 4.0 \%)$ & $5.9( \pm 4.0 \%)$ & K5III & Gordon et al. (2007) \\
\hline 181597 & HR 7341 & $28.0( \pm 3.29 \%)$ & $13.6( \pm 3.34 \%)$ & $5.2( \pm 3.42 \%)$ & K1III & Hammersley et al. (1998) $)^{b}$ \\
\hline 15008 & $\delta$ Hyi & $22.9 \pm 0.8$ & $11.2( \pm 3.5 \%)$ & $4.4( \pm 3.5 \%)$ & $\mathrm{A} 1 / 2 \mathrm{~V}$ & Gordon et al. (2007) \\
\hline 156729 & e Her & $12.0( \pm 3.21 \%)$ & $5.8( \pm 3.25 \%)$ & $2.2( \pm 3.28 \%)$ & $\mathrm{A} 2 \mathrm{~V}$ & Hammersley et al. $(1998)^{b}$ \\
\hline 168009 & HR 6847 & $10.0( \pm 3.40 \%)$ & $4.9( \pm 3.45 \%)$ & $1.9( \pm 3.50 \%)$ & $\mathrm{G} 2 \mathrm{~V}$ & Hammersley et al. (1998) \\
\hline
\end{tabular}

Notes. Source fluxes from Gordon et al. (2007) are for an effective wavelength of $71.42 \mu \mathrm{m}$ and have been colour-corrected to the PACS central wavelength of $70 \mu \mathrm{m}$ by dividing by the factor 0.961 (cf. Müller et al. 2011) for a Rayleigh-Jeans-tail-type SED. 100 and $160 \mu \mathrm{m}$ fluxes for these sources are then extrapolated values for this adopted SED. Source flux models are from ${ }^{(a)} \mathrm{http}: / /$ general-tools. cosmos. esa.int/iso/users/ expl_lib/ISO/wwwcal/isoprep/cohen/extraps/, ${ }^{(b)}$ http://general-tools.cosmos.esa.int/iso/users/expl_lib/ISO/wwwcal/ isoprep/gbpp/.

2) How does the finally achieved sensitivity compare with predictions by the PACS exposure-time calculator of the Herschel observation planning tool?

3) What is the impact and consistency of the applied data reduction scheme on the resulting source flux for fainter and fainter flux contributions on top of the telescope background level?

4) What is the impact of background confusion noise on the resulting fluxes and the sensitivity limit?

Ultimately, some of the faint sources may be characterized well enough to become primary standard sources for future powerful and sensitive FIR space telescopes, such as SPICA (e.g. Sibthorpe et al. 2015), Millimetron (e.g. Smirnov et al. 2012) or the Origins Space Telescope (Meixner et al. 2017).

Most of the observations have been done in mini-scan-map mode, but we have included also a valuable set of complementary chop/nod point-source photometry. We first report the scan map photometry including the sensitivity verification. Then we present the chop/nod photometry and compare it with the scan map results. Finally, we analyse the source spectral energy distributions (SEDs) by comparison with model SEDs and establish which sources are suitable as accurate celestial standards.

\section{Source selection}

In preparation of the PACS in-flight photometric calibration, secondary standard source lists with stars described in Cohen et al. (1996), Hammersley et al. (1998), and Gordon et al. (2007) were prepared by the PACS Instrument Control Centre (ICC) team. Depending on the source visibility during the Herschel mission, a subset of sources from these lists were observed to cover the flux range from $0.5-2.5$ Jy down to $2-10 \mathrm{mJy}$ over the three photometer wavelengths 70,100 , and $160 \mu \mathrm{m}$. The finally observed 17 sources are listed in Table 1.

\section{Scan map photometry}

Fifteen out of the 17 sources were observed in the PACS mini-scan-map point-source observing mode. This was the recommended scientific observing mode for point sources after Herschel's Science Demonstration Phase (SDP), because it had a better sensitivity and allowed a better characterization of the source vicinity and larger-scale structures of the background than chop/nod photometry. The satellite scans were mostly done with the nominal $20^{\prime \prime} / \mathrm{s}$ speed; a few early ones were done with the originally adopted speed of $10^{\prime \prime} / \mathrm{s}$. The scan map dimension parameters are usually $3^{\prime}$ leg length and ten legs with a separation of $4^{\prime \prime}$ with scan angles in array coordinates of $70^{\circ}$ and $110^{\circ}$ (along the diagonal of the bolometer arrays). Only a few early measurements had different parameters from these values, when still probing for the optimum parameter set. In the case of repetition factors larger than 1, in particular for our faintest targets, the whole scan map was repeatedly executed according to the specified factor. We note that a repetition factor may have been optimized for the short wave filter measurement and is hence less optimal for the $160 \mu \mathrm{m}$ filter, where the star is fainter. The observations were usually done in high gain mode. There are a few exceptions taken for comparative performance checks. Selected observing parameters are listed for all individual scan map observations in Tables A.3 to A.5 which are available in electronic format at CDS.

\subsection{Data analysis and calibration}

The data reduction and calibration performed in $\mathrm{HIPE}^{1}$ (Ott 2010) followed the description in Balog et al. (2014), applying

1 HIPE is a joint development by the Herschel Science Ground Segment Consortium, consisting of ESA, the NASA Herschel Science center, and the HIFI, PACS and SPIRE consortia. 
Table 2. Relevant scan map parameters for photometry and noise determination.

\begin{tabular}{rcccccccc}
\hline \hline $\begin{array}{r}\text { Filter } \\
(\mu \mathrm{m})\end{array}$ & $\begin{array}{c}r_{\text {aper }}^{\text {phot }} \\
\left({ }^{\prime \prime}\right)\end{array}$ & $c_{\text {aper }}$ & $c c_{\text {star }}\left(\lambda_{\text {ref }}\right)$ & HPF radius $^{a}$ & Pixfrac & $\begin{array}{c}\text { Outpix } \\
\left({ }^{\prime \prime}\right)\end{array}$ & $N_{\text {aper }}$ & $f_{\text {corr }}$ \\
\hline 70 & 5.6 & 1.61 & 1.016 & 15 & 1.0 & 1.1 & 81.42 & 3.13 \\
100 & 6.8 & 1.56 & 1.033 & 20 & 1.0 & 1.4 & 74.12 & 2.76 \\
160 & 10.7 & 1.56 & 1.074 & 35 & 1.0 & 2.1 & 81.56 & 4.12 \\
\hline
\end{tabular}

Notes. $r_{\text {aper }}^{\text {phot }}$ is the radius of the aperture used for the point-source photometry, $c_{\text {aper }}$ is the corresponding correction factor to scale the flux to its total value, $c c\left(\lambda_{\text {ref }}\right)$ is the colour-correction factor to derive the source flux at the reference wavelength $\lambda_{\text {ref }}$ of the filter (Müller et al. 2011), HPF is the abbreviation for high pass filter, pixfrac is the ratio of drop size to input pixel size used for the drizzling algorithm (Fruchter \& Hook 2002) within the photProject() mapper, outpix is the output pixel size in the final map, $N_{\text {aper }}$ is the number of output pixels inside the photometry aperture with $r_{\text {aper }}^{\text {phot }}$, and $f_{\text {corr }}$ is the correlated noise correction factor depending on the combination of HPF radius / pixfrac / outpix. ${ }^{(a)}$ This parameter determines the elementary section of a scan over which the HPF algorithm computes a running median value. Its unit is "number of read-outs". The spatial interval between two readouts is $\alpha_{\mathrm{ro}}=\frac{v_{\mathrm{scan}}}{v_{\mathrm{ro}}}$. For the standard $v_{\mathrm{ro}}=10 \mathrm{~Hz}$ read-out scheme in PACS prime mode, and a scan speed $v_{\mathrm{scan}}=20^{\prime \prime} / \mathrm{s}$, the spatial interval $\alpha_{\mathrm{ro}}$ between two read-outs corresponds to $2^{\prime \prime}$. The entire width of the HPF window $\left({ }^{\prime \prime}\right)=[(2 \times \operatorname{HPF}$ radius $)+1] \times \alpha_{\mathrm{ro}}$.

the high-pass filter (HPF) algorithm to remove the $\frac{1}{f}$-noise from the scan data of the bolometer detectors. A few recent developments in PACS data reduction (gyro correction and updated pointing products, refined focal plane geometry calibration and more precise timing of the detector readouts) have been included.

The source flux is determined by aperture photometry. The relation between the final stellar flux at the reference wavelength of the respective filter $(70,100$, and $160 \mu \mathrm{m}), f_{\text {star }}\left(\lambda_{\text {ref }}\right)$, and the integrated background subtracted map flux inside the aperture, $f_{\text {aper }}$, is given by

$f_{\text {star }}\left(\lambda_{\text {ref }}\right)=\frac{c_{\text {aper }}\left(\lambda_{\text {ref }}\right) \times f_{\text {aper }}\left(\lambda_{\text {ref }}\right)}{c c_{\text {star }}\left(\lambda_{\text {ref }}\right)}$

where $c_{\text {aper }}$ is the aperture correction factor to get the total non-colour-corrected source flux, $f_{\text {tot }}$. Since the PACS calibration scheme yields a flux related to a SED $v \times f=$ const. the colour-correction factor $c c_{\text {star }}\left(\lambda_{\text {ref }}\right)$ provides the appropriate correction for the stellar SED (5000 K blackbody). The aperture and colour-correction factors are listed in Table 2.

For the investigation of background contamination we also used the JScanam algorithm (Graciá-Carpio et al. 2015), which better preserves extended emission. For the final projection of all data, the HIPE algorithm photProject() was applied; the selected mapping parameters pixfrac and output pixel size are listed in Table 2.

\subsection{Optimum aperture size for faint star photometry}

For the faint star photometry we have selected smaller apertures (cf. Table 2) than were used for the fiducial star photometry in Balog et al. (2014) (12", 12", and 22", respectively). These are the same aperture sizes as for chop-nod photometry.

These smaller apertures, which are adapted to the PSF FWHM in the respective filter, result in a much higher flux reproducibility among the individual measurements and hence a smaller standard deviation of the mean source flux, as well as more reliable and consistent (with regard to the relative spectral shape) source flux measurements for the faintest sources. This is shown in Table A.1 in Appendix A.1, where photometry with the large standard apertures is compared with the photometry applying the smaller apertures. For the cases with $\geq 4$ individual measurements the improvement in reproducibility can be up to a factor of 2-3. The finally achieved average reproducibility
Table 3. Average photometric reproducibility and its standard deviation for the six brightest stars with at least eight individual measurements per filter.

\begin{tabular}{rc}
\hline \hline $\begin{array}{r}\text { Filter } \\
(\mu \mathrm{m})\end{array}$ & $\begin{array}{c}\text { Photometric reproducibility } \\
(\%)\end{array}$ \\
\hline 70 & $0.23 \pm 0.15$ \\
100 & $0.57 \pm 0.63$ \\
160 & $1.85 \pm 1.80$ \\
\hline
\end{tabular}

for sources with eight individual measurements in each filter is listed in Table 3.

From a statistical analysis of the signals of the approximately 21000 PACS photometer internal calibration source measurements, Moór et al. (2014) derived a stability of the PACS bolometer response of about $0.2 \%$ standard deviation or $2 \%$, $3 \%$, and $5 \%$ peak-to-peak at 70,100 , and $160 \mu \mathrm{m}$, respectively, after correction for evaporator temperature effects and initial signal drifts after cooler recycling and photometer switch-on. Our photometry includes the evaporator temperature correction and practically all measurements are outside phases with noticeable initial signal drifts. The mean reproducibility of the $70 \mu \mathrm{m}$ stellar fluxes comes close to the standard deviation of the bolometer response. At 100 and $160 \mu \mathrm{m}$ the mean reproducibility is less good and shows a larger scatter, firstly because the sources are weaker and secondly because the uncertainties in background subtraction are higher.

\subsection{Noise and S/N determination}

A flux histogram has been constructed for all output pixels of the image map, where the corresponding coverage map ${ }^{2}$ indicates that cover $_{\text {pix }} \gtrsim \frac{1}{2}$ cover $_{\text {max }}$. This is justified, since the stars are located in the central part of the map around the highest coverage. A Gauss fit has been performed to the histogram but restricted to the part with fluxes below the bin associated with the maximum number, representing in first approximation the background level, and hence avoiding contamination of the derived

2 The coverage map gives the sum of all complete $(=1.0)$ or partial $(<1.0)$ coverage occurrences of each map output pixel by any physical array pixel, reduced to the specified drop size, from all unmasked readout frames along the scan time-line. 


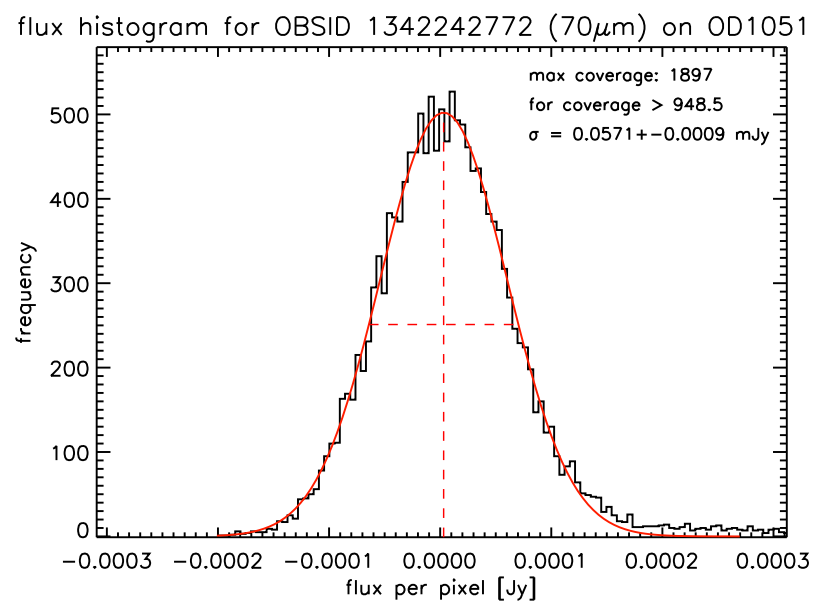

Fig. 1. Illustration of the histogram method to determine the background noise. The example shows the number of pixels per flux bin of the $70 \mu \mathrm{m}$ map of OBSID $1342242772(\beta \mathrm{Gem}$ on OD 1051) for all pixels with a coverage value $>\frac{1}{2}$ cover $_{\max }(>948.5)$. The displayed flux distribution is cut off towards higher fluxes. The red curve is the Gaussian fit to this histogram. For this fit we took all bins left of the distribution maximum into account, but limited the right fitting range to ten bins beyond the distribution maximum in order to avoid a bias of the fitted width by true source flux. The vertical and horizontal red dashed lines indicate the mean background level and the $F W H M=\sqrt{2 \log (2)} \sigma_{\text {pix }}$, respectively. For fluxes per pixel above $\approx 0.12 \mathrm{mJy}$ the contribution by true sources becomes noticeable.

noise per pixel, $\sigma_{\text {pix }}$, by flux of faint sources (to optimize the quality of the fit, actually about 10 bins above the bin with the maximum number are included in the fit). An example of this procedure is shown in Fig. 1. This method provides very reliable and homogeneous noise figures.

For our photometric measurements, the noise inside the measurement aperture must be determined from the noise per pixel $\sigma_{\text {pix }}$. This is given by

$\sigma_{\text {aper }}=\sqrt{N_{\text {aper }}} \times \sigma_{\text {pix }}$,

with $N_{\text {aper }}$ being the number of map output pixels inside the measurement aperture. The respective numbers of $N_{\text {aper }}$ are listed in Table 2.

The high pass filtering and map projection lead to correlated noise which must be corrected to reconstruct the real detector noise (Popesso et al. 2012). This is achieved by applying the correlated noise correction factor $f_{\text {corr }}$

$f_{\text {corr }}=\sum_{0 \leq i+j+k \leq n}^{n=3} c_{i j k} h p f^{i}$ outpix ${ }^{j}$ pixfrac ${ }^{k}$

$k=0, n ; j=0,(n-k) ; i=0,(n-k-j)^{3}$.

Hence, the noise corrected for correlated noise inside the measurement aperture is

$\sigma_{\text {aper,corr }}=\sqrt{N_{\text {aper }}} \times f_{\text {corr }} \times \sigma_{\text {pix }}$.

The $\mathrm{S} / \mathrm{N}$ of the measurement is then determined as

$\frac{S}{N}=\frac{f_{\text {aper }}}{\sigma_{\text {aper,corr }}}$

$3 c_{i j k}$ is related to the 20 parameters $\mathrm{P}(0) \ldots \mathrm{P}(19)$ in Table 9 of Popesso et al. (2012) by running three nested DO-loops with (from outer to inner) $\mathrm{k}=0, \mathrm{n} ; \mathrm{j}=0,(\mathrm{n}-\mathrm{k})$; and $\mathrm{i}=0,(\mathrm{n}-\mathrm{k}-\mathrm{j})$.
Table 4. RMS noise values $f_{1 \sigma, 1 s}$, the $1 \sigma\left(\frac{S}{N}=1\right)$ flux level being achievable with an integration time of $1 \mathrm{~s}$, used in HSpot for $\mathrm{S} / \mathrm{N}$ calculation.

\begin{tabular}{rc}
\hline \hline $\begin{array}{r}\text { Filter } \\
(\mu \mathrm{m})\end{array}$ & $\begin{array}{l}f_{1 \sigma, 1 s} \\
(\mathrm{mJy})\end{array}$ \\
\hline 70 & 30.6 \\
100 & 36.0 \\
160 & 68.5 \\
\hline
\end{tabular}

where $f_{\text {aper }}$ is the part of the source flux measured inside the aperture.

\subsection{The dependence of $S / N$ on exposure time and flux}

The measured S/Ns are compared with the $\mathrm{S} / \mathrm{N}$ predictions of the exposure time calculation tool in the Herschel Observatory Planning Tool HSpot (Herschel-Spot (HSpot) User's Guide: Herschel Observation Planning Tool 2013). HSpot calculates the S/Ns based on an rms noise due to telescope thermal noise emission and the electrical noise of the read-out electronics, cf. Table 4:

$\bar{N}_{\text {HSpot }}=\frac{f_{\text {star }}}{f_{1 \sigma, 1 s}} \sqrt{n_{\text {rep }} t_{\text {obscover }}}$,

with $f_{\text {star }}$ using the colour- and aperture-corrected total stellar flux.

This S/N scales with the square root of the coverage time of the source during one scan map, $t_{\text {obscover }}$ and the number of scan map repetitions, $n_{\text {rep }}$. For mini-scan-maps, $t_{\text {obscover }}$ is maximum at the map centre and decreases towards the boarders. In analogy to the noise determination in the final maps, as described in Sect. 3.3, we use $t_{\text {obscover }}=\frac{1}{2} t_{\text {obscovercent }}$. The value of $t_{\text {obscover }}$ depends on the scan map parameters (scan leg length, scan leg separation and number of scan legs) and is listed in Table 5 for all scan map parameter combinations used for our observations.

Figure 2 shows the dependence of the achieved S/Ns on time, represented as number of scan repetitions, and the comparison with the HSpot prediction. This includes combined maps of scan and cross-scans, which have the sum of the scan repetitions of the individual maps.

For 70 and $100 \mu \mathrm{m}$ measurements we find $S / N \propto \sqrt{n_{\text {rep }}}$. There are deviations from this relation in that respect that the $\mathrm{S} / \mathrm{N}$ of the combined maps is higher than the expected factor of $\sqrt{2}$ by a few percent. The ratio of the average measured $\mathrm{S} / \mathrm{N}$ to the HSpot prediction is $1.14-1.22$ at $70 \mu \mathrm{m}$ and $1.03-1.09$ at $100 \mu \mathrm{m}$, respectively. Given the fact that the HSpot prediction is for half maximum coverage and the noise determination in the maps is above a threshold of half maximum coverage, the measured $\mathrm{S} / \mathrm{N}$ can be considered as consistent with the HSpot prediction.

For the $160 \mu \mathrm{m}$ measurements we find for small repetition numbers $\left(n_{\text {rep }} \leq 12\right)$ that the $\mathrm{S} / \mathrm{N}$ increases with the $\sqrt{n_{\text {rep }}}$ for single and combined maps. For higher repetition numbers it is obvious that the increase of the measured S/Ns is flatter. This flattening is caused by confusion noise, which will be discussed in the following Section. The ratio of the average measured $\mathrm{S} / \mathrm{N}$ to the HSpot prediction is around 0.80 . We note, however, that there is some margin in achievable $\mathrm{S} / \mathrm{N}$ depending on the selection of the HPF radius. We calculate a decrease of the resulting noise by $\approx 23 \%$ between HPF radius $=40$ read-outs and $\mathrm{HPF}$ radius $=15$ read-outs for pixfrac $=1.0$ and output 
Table 5. Central coverage time of a source during a scan map execution depending on the scan map parameters (scan leg length, scan leg separation and number of scan legs) as calculated by HSpot.

\begin{tabular}{ccr}
\hline $\begin{array}{c}\text { Scan map parameters } \\
\left({ }^{\prime \prime} /{ }^{\prime \prime} / \#\right)\end{array}$ & $\begin{array}{c}\text { Map angle } \\
(\mathrm{deg})\end{array}$ & $\begin{array}{r}t_{\text {obscovercent }} \\
(\mathrm{s})\end{array}$ \\
\hline \multicolumn{3}{c}{ scan speed $20^{\prime \prime} / \mathrm{s}$} \\
$150 / 4 / 10$ & $70 / 110$ & 80 \\
$\mathbf{1 8 0 / 4} / \mathbf{1 0}$ & $70 / 110$ & 90 \\
$210 / 4 / 20$ & 90 & 220 \\
$210 / 4 / 25$ & 90 & 275 \\
$240 / 4 / 8$ & $63 / 117$ & 96 \\
$90 / 5 / 9$ & $70 / 110$ & 45 \\
$210 / 15 / 4$ & 90 & 44 \\
\hline \multicolumn{3}{c}{ scan speed $10^{\prime \prime} / \mathrm{s}$} \\
\hline $120 / 3 / 21$ & $80 / 100$ & 252 \\
$150 / 4 / 9$ & $85 / 95$ & 144 \\
$210 / 4 / 20$ & 90 & 440 \\
$210 / 4 / 25$ & 90 & 550 \\
$90 / 5 / 9$ & $60 / 120$ & 90 \\
$120 / 5 / 9$ & $80 / 100$ & 108 \\
$210 / 15 / 4$ & 90 & 88 \\
\hline
\end{tabular}

Notes. The combination in bold face is the default combination used for the majority of the measurements.

pixel size of 2'. 0 according to the formalism in Popesso et al. (2012). The latter harsh filter width would only be applied for maps with very weak sources covering only a few pixels. The HSpot values were derived from cosmological fields, where harsh HPF filter widths could be applied, and that would explain the somewhat worse performance for our milder HPF radius of 35 read-outs.

After OD 1375, half of the red photometer array was lost. We have one good case, namely the $160 \mu \mathrm{m}$ observations of $\varepsilon$ Lep from OD 1377 , to check the performance relative to full array observations. With regard to comparable observations from ODs 502, 833, and 1034 (cf. Table A.5 available in electronic form at CDS), we find the following: The coverage is 0.51 of the full array map, but the noise is increased by only a factor of 1.21.

In the case of $\eta$ Dra the performance of scan speeds $10^{\prime \prime} / \mathrm{s}$ and $20^{\prime \prime} / \mathrm{s}$ can be inter-compared. While the coverage time of the $10^{\prime \prime} / \mathrm{s}$ scan speed maps is always greater than or equal to twice the coverage time of the $20^{\prime \prime} / \mathrm{s}$ scan speed (cf. configurations in Tables A.3 to A.5, available in electronic form at CDS and corresponding coverage times in Table 5), the measured $\mathrm{S} / \mathrm{N}$ of the $20^{\prime \prime} / \mathrm{s}$ scan speed is above the HSpot prediction at 70 and $100 \mu \mathrm{m}$, while the measured S/Ns of all $10^{\prime \prime} / \mathrm{s}$ scan speed combinations are below the HSpot prediction. This is a clear demonstration that the $20^{\prime \prime} / \mathrm{s}$ scan speed maps are relatively more sensitive than their $10 " / \mathrm{s}$ scan speed counterparts. At $160 \mu \mathrm{m}$ this is even more pronounced; the $\mathrm{S} / \mathrm{N}$ of the $20^{\prime \prime} / \mathrm{s}$ scan speed map with half of the coverage time is better than that of the $10^{\prime \prime} / \mathrm{s}$ scan speed map with otherwise identical map parameters.

Figure 3 shows the dependence of the achieved S/Ns on flux. For the 70 and $100 \mu \mathrm{m}$ filters we can verify that the $\mathrm{S} / \mathrm{N}$ scales linearly with flux over two decades of flux and at least down to total (aperture corrected) source fluxes of $30 \mathrm{mJy}$ and $18 \mathrm{mJy}$, respectively. For the $160 \mu \mathrm{m}$ filter the linearity with flux can be verified over about one decade in flux down to a total (aperture corrected) source flux of $85 \mathrm{mJy}$ for repetition factors $1-12$. For fainter fluxes measurements with higher $(\geq 20)$ repetition factors
Table 6. S/N determination at $160 \mu \mathrm{m}$ for HD 181597 and HD 15008 , which are below the detection limit $(S / N \lesssim 1.5)$ at this wavelength.

\begin{tabular}{rcccc}
\hline \hline $\begin{array}{r}\mathrm{HD} \\
(\mu \mathrm{m})\end{array}$ & $\begin{array}{r}f_{\text {predict }} \\
(\mathrm{mJy})\end{array}$ & $\begin{array}{c}f_{\text {aper }} \\
(\mathrm{mJy})\end{array}$ & $\begin{array}{c}\sigma_{\text {aper,corr }} \\
(\mathrm{mJy})\end{array}$ & $\frac{S}{N}$ \\
\hline 181597 & 5.2 & 3.3 & 2.6 & 1.3 \\
15008 & 4.4 & 2.8 & 3.2 & 0.9 \\
\hline
\end{tabular}

are necessary to achieve a $\mathrm{S} / \mathrm{N}$ which is sufficiently above values close to the detection limit $(S / N \lesssim 1.5$, cf. Sect. 3.6). These high repetition factors give an increase in $\mathrm{S} / \mathrm{N}$ that is smaller than expected, which we explain in the following Section as being due to a confusion noise contribution. Since the confusion noise contribution is not the same in the different source fields, the linearity of the $\mathrm{S} / \mathrm{N}$ with flux cannot be verified any more straightforwardly in the $160 \mu \mathrm{m}$ flux range below $85 \mathrm{mJy}$.

\subsection{Impact on $S / N$ by background confusion noise}

In particular at $160 \mu \mathrm{m}$, there may be another relevant noise source, which is FIR background confusion noise. This is composed of a cosmic infrared background component and a galactic cirrus component. Examples of background confusion, which also affects the source photometry, are shown in Figs. 5 and 6. The confusion noise is independent of on-source observation time, that is, in the case of approaching the confusion noise limit, the $\mathrm{S} / \mathrm{N}$ does not improve anymore with on-source observation time.

$$
\frac{S}{N}{ }_{\text {HSpot,conf }}=\frac{f_{\text {star }}}{\sqrt{\frac{f_{1 \sigma, 1 s}^{2}}{n_{\text {rep }} t_{\text {obscover }}}+f_{\text {confnoise }}^{2}}} .
$$

This leads to the effect that the $\mathrm{S} / \mathrm{N}$ curve with time flattens out as observed for the $160 \mu \mathrm{m} \mathrm{S} / \mathrm{Ns}$ in Fig. 2. For our source fields, HSpot returns a $160 \mu \mathrm{m}$ point source equivalent confusion noise estimate $f_{\text {confnoise }}$ between 1.3 and $1.5 \mathrm{mJy}$. The typical onsource observation time for repetition factor 1 is $45 \mathrm{~s}$, resulting in a $160 \mu \mathrm{m}$ noise level of $10 \mathrm{mJy}$. This is about a factor of 7 higher than the estimated confusion noise and only for large scan map repetition factors $(>10)$, does the confusion noise become significant.

\subsection{Detection limits}

HD 181597 and HD 15008 ( $\delta$ Hyi) are good examples for nondetections at $160 \mu \mathrm{m}$, because the expected source flux is below the detection limit. Both sources have a clear detection at $70 \mu \mathrm{m}$ ( $S / N=30$ and 15 , respectively), which allows to identify the expected source position on the $160 \mu \mathrm{m}$ maps (see Fig. 4). Table 6 lists the determined S/Ns, which are $<1.5$ (we note, that the $\mathrm{S} / \mathrm{N}$ measurement in the map is actually higher by the factor $f_{\text {corr }}=4.12$, which corresponds to the classic $\mathrm{S} / \mathrm{N}$ detection limit of 5-6). This is in accordance that no source can be detected at the location of the star in the $160 \mu \mathrm{m}$ map.

\subsection{Confusion by neighboring sources and cirrus}

The fainter the star, the higher the probability, in particular at 100 and $160 \mu \mathrm{m}$, that nearby sources confuse the source flux inside the measurement aperture.

A clear case of confusion by neighboring sources is shown in Fig. 5 for the star HD 159330. While at $70 \mu \mathrm{m}$ the star is more 

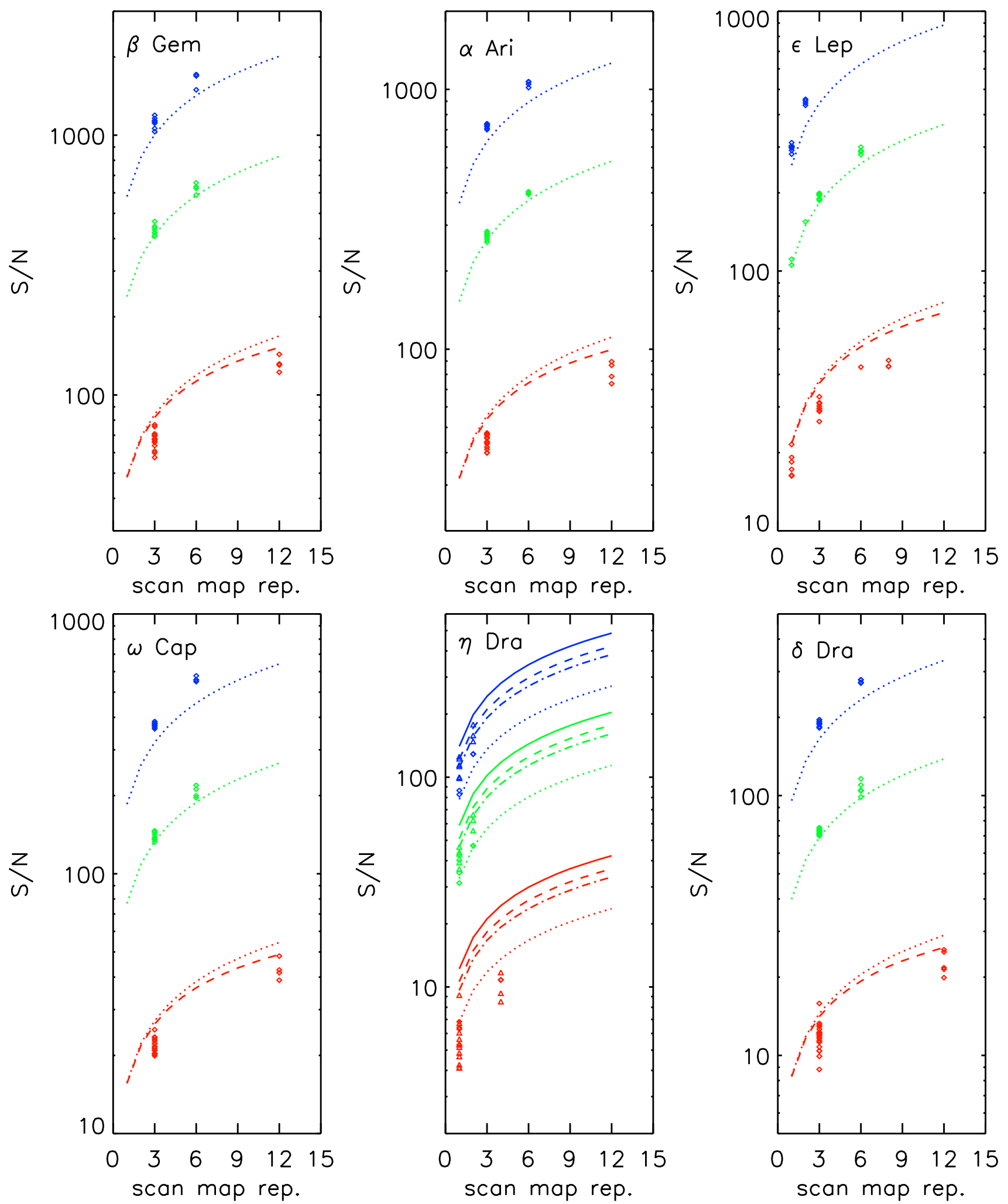

Fig. 2. Measured S/Ns for mini-scan-map photometry depending on the number of repetitions. Blue, green, and red symbols represent measurements in the three filters, 70,100 , and $160 \mu \mathrm{m}$, respectively. Diamond symbols indicate a scan speed of $20^{\prime \prime} / \mathrm{s}$, triangles a scan speed of $10^{\prime \prime} / \mathrm{s}$. The dotted lines in the respective colours show the S/N prediction by the PACS exposure time calculator of the Herschel observation planning tool HSpot for the measured colour corrected stellar flux. Long dashed red lines indicate the S/N prediction including confusion noise. An exception is the panel of $\eta$ Dra, where the sets of four dotted, dashed, and dashed-dotted lines represent the sensitivity predictions for four different map parameter combinations; the upper three are with $10^{\prime \prime} / \mathrm{s}$ scan speed, the lowest one is with $20^{\prime \prime} / \mathrm{s}$ scan speed. For more details, see text. 
U. Klaas et al.: Herschel-PACS photometry of faint stars
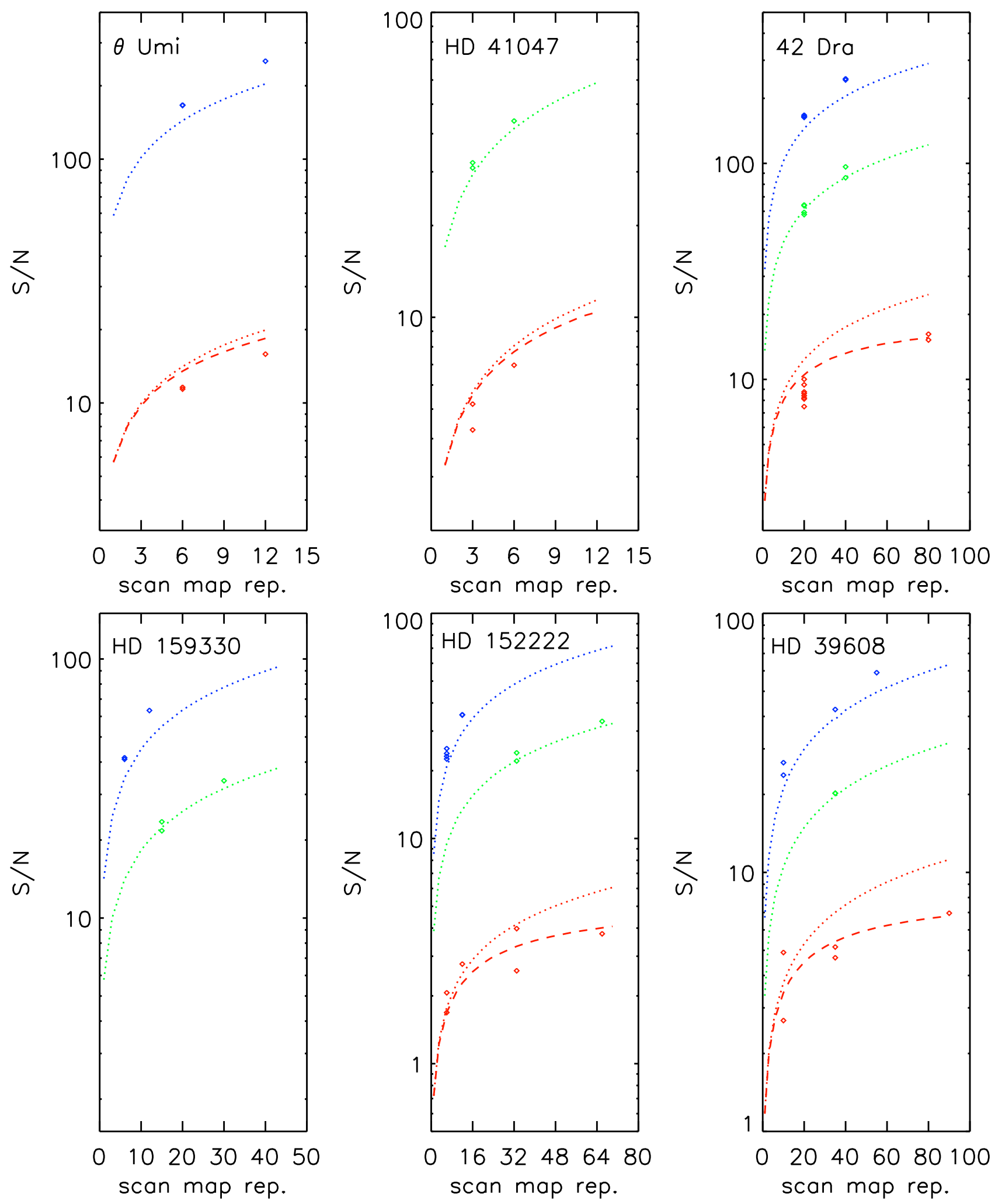

Fig. 2. continued. Measured S/Ns for mini scan map photometry depending on the number of repetitions.

or less the only visible source inside the field, at $100 \mu \mathrm{m}$ a small cluster of sources around the star starts to pop up, but the star is still the dominant source inside the field. At $160 \mu \mathrm{m}$, all surrounding sources are brighter than the star, which appears only as an appendix of the source located north-west of it. Also the local brightness maximum is not as well located on the cross as is the case for the stellar images at 70 and $100 \mu \mathrm{m}$. It is therefore not possible to obtain reliable photometry for HD 159330 at $160 \mu \mathrm{m}$. The compactness of the surrounding sources both in the HPF and the JScanam image points to an extragalactic nature of the confusing sources. This is difficult to verify in the optical, since HD 159330 is a $6.2 \mathrm{mag}$ ( $V$ band) bright star.

An example of likely cirrus confusion is shown in Fig. 6 around the star $\eta$ Dra (HD 148387). There is relatively significant 

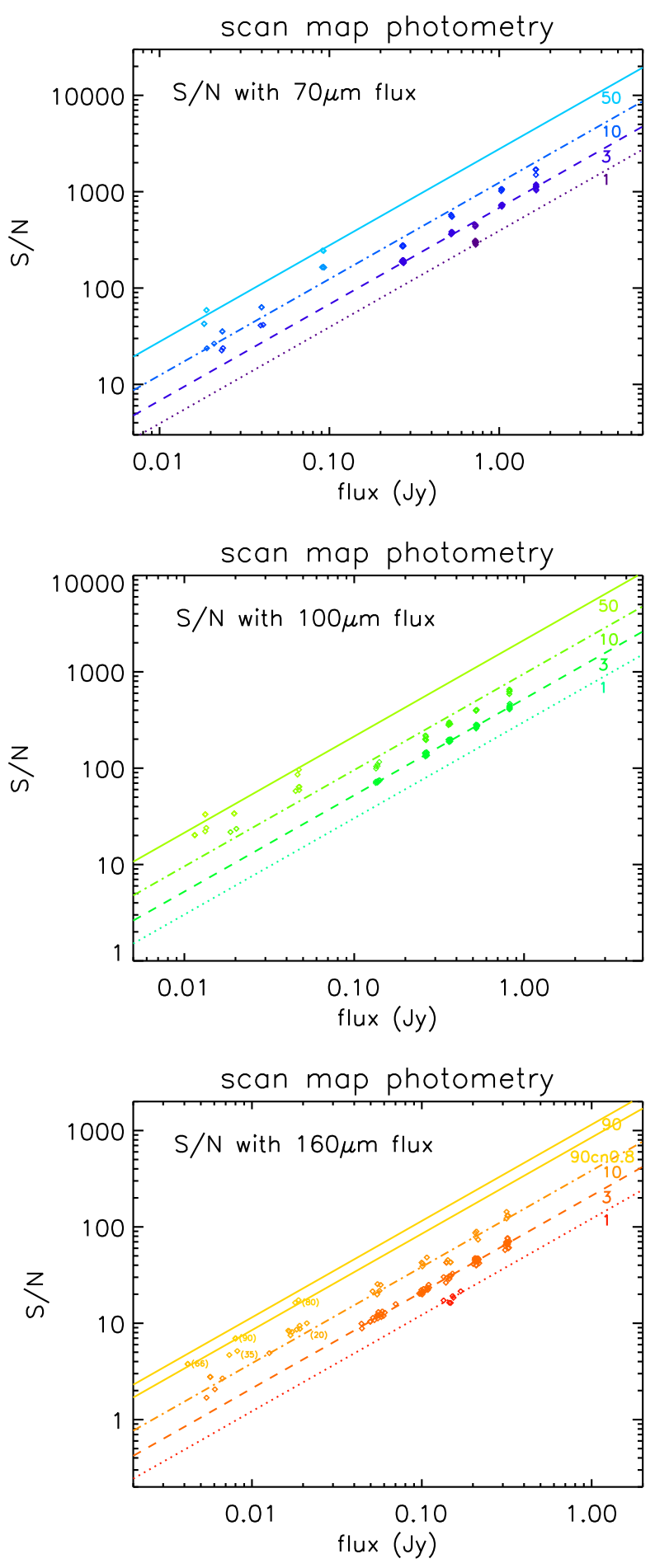

Fig. 3. Measured S/Ns for mini-scan-map photometry depending on the source flux (Note: fluxes measured inside the aperture are used here). For better comparability only measurements with an observational setup identical with the final mini-map set-up (ten 180" scan legs with $4^{\prime \prime}$ separation and scan speed 20"/s) are considered. Lighter colour tones are measurements with higher scan map repetition factors. We note that here the dotted, dashed, and dashed dotted lines in different colour tones do not represent the $\mathrm{S} / \mathrm{N}$ prediction by the PACS exposure time calculator of the Herschel observation planning tool HSpot, but are empirical adjustments to the average measured $\mathrm{S} / \mathrm{N}$ for the respective scan map repetitions. In the $160 \mu \mathrm{m}$ panel, numbers in parentheses mark measurements with high repetition factors whose $\mathrm{S} / \mathrm{N}$ is degraded by confusion noise. This is also indicated by two $\mathrm{S} / \mathrm{N}$ with flux lines for repetition factor 90 , where the lower one includes additional confusion noise $(\mathrm{cn})$ of $0.8 \mathrm{mJy}$. similarity between the HPF and JScanam processed map concerning the brighter spots and features, while on the low level there are differences, because the HPF algorithm is not designed to preserve faint extended emission. Nevertheless both maps indicate a filamentary emission around the star. Indeed, $\eta$ Dra, with $1=92.6^{\circ}$ and $\mathrm{b}=+41.0^{\circ}$, is located at the edge of the Draco nebula (cf. e.g. Fig. 3 in Herbstmeier et al. 1998, w.r.t. its location), a pronounced cirrus cloud. The extract of the AKARI Wide-L $(140 \mu \mathrm{m})$ all sky map ${ }^{4}$ (Doi et al. 2015) reveals that $\eta$ Dra is located at the southern edge of a cirrus knot with an extension of it passing north-west into the PACS map area. The cirrus confusion affects the derived $160 \mu \mathrm{m}$ flux noticeably, as is discussed in Sect. 5.2. Other cases of suspected cirrus confusion are also discussed there.

\subsection{Photometry results}

Results of individual photometric measurements are given in Appendix A, Sect. A.3 in Tables A.3 to A.5, which are available in electronic form at CDS. Here we report the combined aperture and colour-corrected photometry of all measurements in Table 7. This is identical with the phot_s photometry in Table A.1. The quoted uncertainties of the measurements in Table 7 include the absolute calibration uncertainty of $5 \%$, due to the fiducial star model uncertainties, which is quadratically added to the rms of the mean flux value as quoted in Table A.1.

For 11 stars we obtain reliable photometry in all three PACS bands. There is no $70 \mu \mathrm{m}$ flux for HD 41047, since there are only measurements in the 100 and $160 \mu \mathrm{m}$ filters. There is no $160 \mu \mathrm{m}$ detection for HD 159330 because of confusion noise. For HD 181597 and HD 15008 we obtain reliable fluxes only at $70 \mu \mathrm{m}$, since there are no $100 \mu \mathrm{m}$ scan map measurements and at $160 \mu \mathrm{m}$ the stars are too faint for the applied repetition numbers. Faintest fluxes, for which the photometry has still good quality (accuracy $\leq 15 \%$ ), are about 10-20 mJy.

In Appendix A.2, we conduct a qualitative inter-comparison of the HPF photometry with three other Herschel mapper softwares for HD 152222, the faintest star at $160 \mu \mathrm{m}$ with reliable photometry in all three filters. Aspects like noise behaviour and shape of the intensity profiles are investigated and discussed. The main conclusion is that for the other mappers adapted aperture correction factors need to be established which will be determined on the basis of the high $\mathrm{S} / \mathrm{N}$ fiducial standard star observational database in a forthcoming paper (Balog et al., 2018, in prep.).

The evaluation of the correspondence with the models is done in Sect. 5.

\section{Chop-nod photometry}

\subsection{Observations}

Thirteen out of the 17 sources were observed in the PACS chop-nod point source observing mode. This was the originally recommended PACS photometer observing mode for point and compact sources. This mode used the PACS chopper to move the source on-array by about $50^{\prime \prime}$, corresponding to the size of about one blue/green bolometer matrix (16 pixels) or

\footnotetext{
4 AKARI Far-infrared All-Sky Survey maps query service http://www.ir.isas.jaxa.jp/AKARI/Archive/Images/FIS_ AllSkyMap/search/
}

We use the AKARI WIDE-L $(140 \mu \mathrm{m})$ maps instead of the N160 $(160 \mu \mathrm{m})$ maps, because the latter ones do not have sufficient $\mathrm{S} / \mathrm{N}$ over the whole field for illustration of the background structure. 

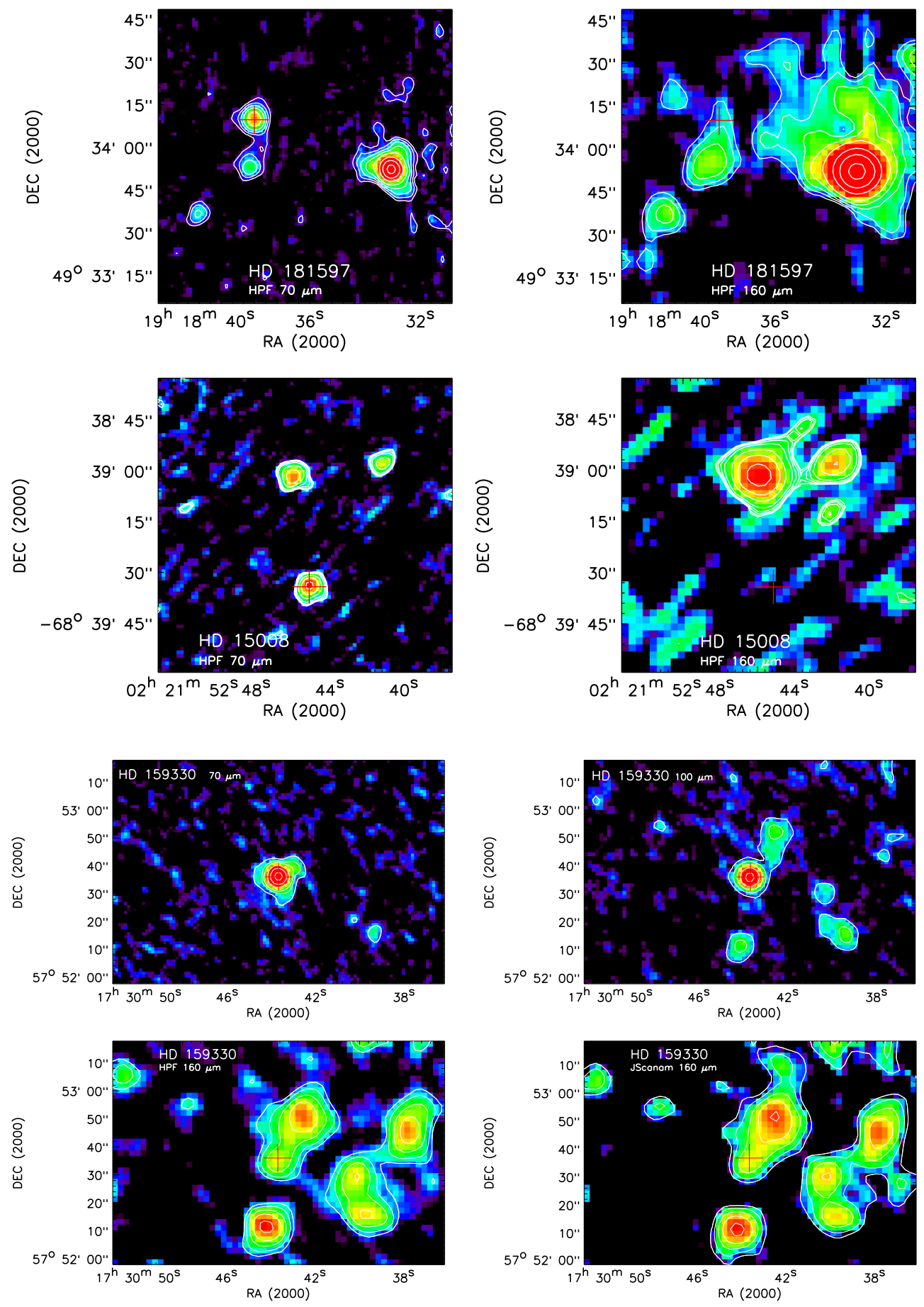

Fig. 4. Examples of detection at $70 \mu \mathrm{m}$ and non-detection at $160 \mu \mathrm{m}$. The red cross (arm length equal to $5^{\prime \prime}$ ) indicates the common Herschel position. We note that for both sources there exists only one scan map orientation which leads to some residual scan artefacts, in vertical and diagonal direction, respectively; see Table A.3, available in electronic form at CDS, for details. Top: HD 181597 , OBSID 1342185451 on OD 146. The bright point source at the right is KIC 11555225. Bottom: HD 15008 $(\delta$ Hyi $), \quad$ OBSID 1342189130 on OD 241.

Fig. 5. Example of background confusion noise around the star HD 159330 (OBSIDs 134221358386 on OD 628, see Tables A.3 and A.4, available in electronic form at CDS, for details) by comparing maps in the three filters 70,100 , and $160 \mu \mathrm{m}$. At $160 \mu \mathrm{m}$ both the high-pass filtered and the JScanam maps are shown to explore the nature of the background sources. The red cross (arm length equal to $5^{\prime \prime}$ ) indicates the best common Herschel position of the star after frame centering at RA $=17: 30: 43.69$ and DEC $=+57: 52: 36.0$. the size of about half a red matrix (8 pixels), with a chopper frequency of $1.25 \mathrm{~Hz}$. The nodding was performed by a satellite movement of the same amplitude but perpendicular to the chopping direction. On each nod position the chopper executed $3 \times 25$ chopper cycles. The three sets of chopper patterns were either on the same array position (no dithering) or on three different array positions (dither option). In the latter case the chopper pattern was displaced parallel to the chopper deflection by 8.15 ( $2 \frac{2}{3}$ blue pixels or $1 \frac{1}{3}$ red pixels). Most of the faint star observations were performed with the dither option on; Tables B.1 to B.3 (available in electronic form at CDS) indicate for each observation the selection of the respective dither/nodither mode. Each chopper plateau lasted for $0.4 \mathrm{~s}$ (16 readouts on-board) producing four frames per plateau in the telemetry down-link. The full $3 \times 25$ chopper cycles per nod position were completed in less than 1 minute. In the case of repetition factors larger than 1 , in particular for our faintest targets, the nod-cycles were repeated in the following way (example for 4 repetitions): nodA-nodB-nodB-nodA-nodA-nodB-nodB-nodA to minimize satellite slew times. Selected repetition factors are given in Tables B.1 to B.3 (available in electronic form at CDS). We note that a repetition factor may have been optimized for the short-wave filter measurement and is hence less optimal for the $160 \mu \mathrm{m}$ filter, where the star is fainter. The observations were usually done in high gain mode, but there were a few exceptions taken for comparative performance checks. 

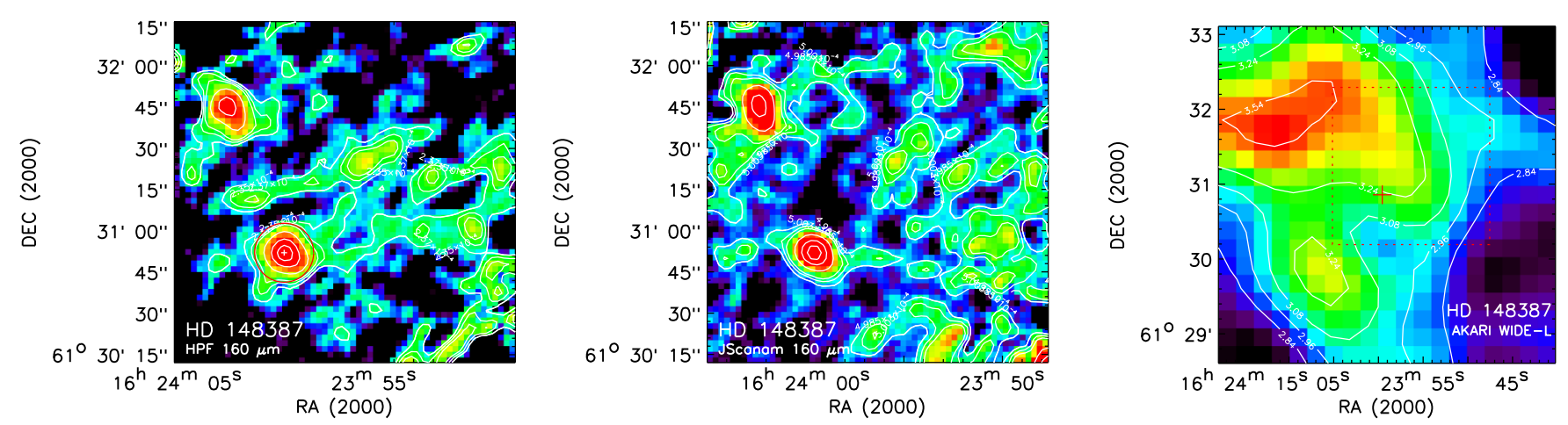

Fig. 6. Example of cirrus confusion noise around the star HD 148387 ( $\eta$ Dra, OBSIDs 1342186146, ..47, ..55, ..56 from OD 160). The left panel shows the HPF processed map used for the photometry, the photometric aperture with 10 ! 7 radius is indicated by the red circle and a small white cross at its centre. The middle panel shows the Jscanam processed map which reproduces extended emission more reliably. The right panel shows the AKARI WIDE-L $(140 \mu \mathrm{m})$ background emission around the source (red cross), the AKARI-map area is about four times as large as the PACS map area, which is indicated by the red dashed square.

Table 7. Combined mini-scan-map photometry.

\begin{tabular}{|c|c|c|c|c|c|c|c|c|c|c|}
\hline HD & Name & $n_{70}$ & $\begin{array}{c}f_{\text {star }}(70 \mu \mathrm{m}) \\
(\mathrm{mJy})\end{array}$ & $\frac{f_{\text {star }}(70 \mu m)}{f_{\text {model }}}$ & $n_{100}$ & $\begin{array}{c}f_{\text {star }}(100 \mu \mathrm{m}) \\
(\mathrm{mJy})\end{array}$ & $\frac{f_{\text {star }}(100 \mu m)}{f_{\text {model }}}$ & $n_{160}$ & $\begin{array}{c}f_{\text {star }}(160 \mu \mathrm{m}) \\
(\mathrm{mJy})\end{array}$ & $\frac{f_{\text {star }}(160 \mu m)}{f_{\text {model }}}$ \\
\hline 62509 & $\beta \mathrm{Gem}$ & 8 & $2649 \pm 132$ & $1.08 \pm 0.08$ & 8 & $1284 \pm 64$ & $1.08 \pm 0.08$ & 16 & $497 \pm 25$ & $1.09 \pm 0.08$ \\
\hline 12929 & $\alpha$ Ari & 8 & $1664 \pm 83$ & $0.97 \pm 0.08$ & 8 & $820 \pm 41$ & $0.99 \pm 0.08$ & 16 & $328 \pm 17$ & $1.02 \pm 0.08$ \\
\hline 32887 & $\varepsilon$ Lep & 8 & $1166 \pm 58$ & $0.99 \pm 0.08$ & 12 & $568 \pm 28$ & $0.99 \pm 0.08$ & $18^{a}$ & $224 \pm 11$ & $1.00 \pm 0.08$ \\
\hline 198542 & $\omega \mathrm{Cap}$ & 8 & $845 \pm 42$ & $0.99 \pm 0.08$ & 8 & $413 \pm 21$ & $0.99 \pm 0.08$ & 16 & $161 \pm 8.2$ & $0.99 \pm 0.08$ \\
\hline 148387 & $\eta$ Dra & 8 & $506 \pm 25$ & $1.06 \pm 0.06$ & 8 & $250 \pm 13$ & $1.07 \pm 0.06$ & 16 & $98 \pm 7.1$ & $1.10 \pm 0.08$ \\
\hline 180711 & $\delta$ Dra & 12 & $436 \pm 22$ & $1.02 \pm 0.08$ & 10 & $214 \pm 11$ & $1.03 \pm 0.08$ & 22 & $85 \pm 4.8$ & $1.07 \pm 0.08$ \\
\hline 139669 & $\theta \mathrm{Umi}$ & 4 & $284 \pm 14$ & $0.99 \pm 0.08$ & 2 & $144 \pm 8.8$ & $1.03 \pm 0.08$ & $2^{b}$ & $62 \pm 4.0^{f}$ & $1.16 \pm 0.10$ \\
\hline 41047 & HR 2131 & - & \multicolumn{2}{|c|}{ no measurement } & 2 & $97 \pm 4.9$ & $1.01 \pm 0.08$ & 2 & $36 \pm 5.9$ & $0.97 \pm 0.17$ \\
\hline 170693 & 42 Dra & 4 & $148 \pm 7.4$ & $0.96 \pm 0.06$ & 4 & $73 \pm 3.8$ & $0.97 \pm 0.06$ & 8 & $28 \pm 1.7$ & $0.96 \pm 0.07$ \\
\hline 138265 & HR 5755 & 4 & $113 \pm 5.7$ & $0.97 \pm 0.06$ & 6 & $57 \pm 2.9$ & $0.99 \pm 0.06$ & $5^{c}$ & $31 \pm 1.8$ & $1.40 \pm 0.10$ \\
\hline 159330 & HR 6540 & 4 & $65 \pm 3.4$ & $1.01 \pm 0.06$ & 6 & $31 \pm 1.9$ & $0.99 \pm 0.07$ & 10 & source co & afusion \\
\hline 152222 & - & 4 & $39 \pm 2.1$ & $0.99 \pm 0.07$ & 2 & $21 \pm 1.0$ & $1.08 \pm 0.07$ & $4^{d}$ & $7.4 \pm 0.9$ & $0.99 \pm 0.13$ \\
\hline 39608 & - & 3 & $31 \pm 1.9$ & $0.99 \pm 0.07$ & 1 & $18 \pm 1.3$ & $1.19 \pm 0.08$ & $2^{e}$ & $12 \pm 0.9$ & $2.05 \pm 0.17$ \\
\hline 181597 & HR 7341 & 2 & $29 \pm 2.5$ & $1.04 \pm 0.09$ & - & \multicolumn{2}{|c|}{ no measurement } & 2 & \multicolumn{2}{|c|}{ below detection limit } \\
\hline 15008 & $\delta$ Hyi & 1 & $22 \pm 1.6$ & $0.96 \pm 0.08$ & - & \multicolumn{2}{|c|}{ no measurement } & 1 & \multicolumn{2}{|c|}{ below detection limit } \\
\hline
\end{tabular}

Notes. Model fluxes are listed in Table 1 . The quoted uncertainties of the measurements include the absolute calibration uncertainty of $5 \%$. In the case of only one observation for a specific source, the statistical error of this flux measurement is used in the uncertainty determination, and in the case of more than one observation for a source, as given in columns $n_{70}, n_{100}$, and $n_{160}$, the standard deviation of the weighted mean as given in Table A.1, column phot_s, is used in the uncertainty calculation. ${ }^{(a)}$ OBSIDs $1342205202 \& 1342263904$ excluded , ${ }^{(b)}$ OBSIDs 1342184574 , $1342184575,1342184585,1342184586$ excluded, ${ }^{(c)}$ OBSIDs 1342185446, 1342185448, 1342185447, 1342185449, 1342191986 excluded, ${ }^{(d)}$ OBSIDs $1342240702,1342240703,1342227973 \& 1342227974,{ }^{(e)}$ Only OBSIDs $1342198537 \& 1342198538,{ }^{(f)}$ Due to cirrus confusion, a background subtraction uncertainty of $10 \%$ must be added: $62 \pm 7.1 \mathrm{mJy}$, see discussion in Sect. 5.2.

\subsection{Data analysis and calibration}

The data reduction and calibration performed in HIPE (Ott 2010) followed the description in Nielbock et al. (2013). For the reduction of our faint star targets we adjusted a few aspects and used very recent software developments for PACS photometer observations (gyro correction and updated pointing products and refined focal plane geometry calibration). These new corrections are meanwhile part of the Standard Product Generation (SPG) pipelines version 13.0 and higher. For the stellar flux derivation the same procedures and parameters as for scan map photometry, and as summarized in Eq. (1) and Table 2, are applied.

The photometric uncertainty was estimated with the histogram method with a coverage threshold as described in detail in Sect. 3.3 for the scan maps. Correlated noise is corrected for via an empirical function to obtain a conservative upper limit for the measurement uncertainties. The applied correction factors $f_{\text {corr }}$ are $6.33,4.22$, and 7.81 for the 70,100 , and $160 \mu \mathrm{m}$ filters, respectively.

\subsection{Photometry results}

Results of individual measurements are given in Appendix B in Tables B.1 to B.3. We note that there are observations of HD 138265 on OD 146 for which the noise does not seem to scale with the number of repetitions. The reason is that for these measurements the basic length of the nod period was varied and compensated by the corresponding number of nod cycle repetitions.

Here we report the combined photometry of all measurements in Table 8. The quoted uncertainties of the measurements 
Table 8. Combined chop-nod photometry.

\begin{tabular}{|c|c|c|c|c|c|c|c|c|c|c|}
\hline HD & Name & $n_{70}$ & $\begin{array}{c}f_{\text {star }}(70 \mu \mathrm{m}) \\
(\mathrm{mJy})\end{array}$ & $\frac{f_{\text {star }}(70 \mu \mathrm{m})}{f_{\text {model }}}$ & $n_{100}$ & $\begin{array}{c}f_{\text {star }}(100 \mu \mathrm{m}) \\
(\mathrm{mJy})\end{array}$ & $\frac{f_{\text {star }}(100 \mu \mathrm{m})}{f_{\text {model }}}$ & $n_{160}$ & $\begin{array}{c}f_{\text {star }}(160 \mu \mathrm{m}) \\
(\mathrm{mJy})\end{array}$ & $\frac{f_{\text {star }}(160 \mu \mathrm{m})}{f_{\text {model }}}$ \\
\hline 62509 & $\beta \mathrm{Gem}$ & 1 & $2570 \pm 129$ & $1.05 \pm 0.08$ & 1 & $1267 \pm 65$ & $1.07 \pm 0.08$ & 2 & $497 \pm 25$ & $1.09 \pm 0.08$ \\
\hline 32887 & $\varepsilon$ Lep & 2 & $1181 \pm 59$ & $1.00 \pm 0.08$ & 2 & $558 \pm 31$ & $0.97 \pm 0.08$ & 4 & $205 \pm 12$ & $0.92 \pm 0.08$ \\
\hline 148387 & $\eta$ Dra & 1 & $509 \pm 31$ & $1.06 \pm 0.07$ & 1 & $236 \pm 19$ & $1.01 \pm 0.10$ & 2 & $101 \pm 10$ & $1.13 \pm 0.10$ \\
\hline 180711 & $\delta$ Dra & 5 & $440 \pm 22$ & $1.03 \pm 0.08$ & 3 & $214 \pm 12$ & $1.03 \pm 0.08$ & 8 & $83 \pm 5.0$ & $1.05 \pm 0.08$ \\
\hline 139669 & $\theta \mathrm{Umi}$ & 10 & $282 \pm 14$ & $0.98 \pm 0.08$ & 9 & $142 \pm 7.9$ & $1.02 \pm 0.08$ & $16^{a}$ & $55 \pm 3.8$ & $1.02 \pm 0.09$ \\
\hline 41047 & HR 2131 & - & - & - & 1 & $88 \pm 5.6$ & $0.92 \pm 0.09$ & 1 & $29 \pm 15$ & $0.77 \pm 0.54$ \\
\hline 138265 & HR 5755 & 9 & $113 \pm 5.7$ & $0.97 \pm 0.06$ & 4 & $59 \pm 3.0$ & $1.04 \pm 0.06$ & $11^{a}$ & $31 \pm 1.9$ & $1.39 \pm 0.07$ \\
\hline 159330 & HR 6540 & 1 & $60 \pm 7.6$ & $0.94 \pm 0.13$ & 3 & $31 \pm 2.2$ & $0.98 \pm 0.08$ & 4 & \multirow{2}{*}{\multicolumn{2}{|c|}{$\begin{array}{l}\text { source confusion } \\
\text { below detection limit }\end{array}$}} \\
\hline 152222 & - & 1 & $39 \pm 4.9$ & $0.98 \pm 0.14$ & - & \multicolumn{2}{|c|}{ no measurement } & 1 & & \\
\hline 181597 & HR 7341 & 1 & \multicolumn{2}{|c|}{ below detection limit } & - & \multicolumn{2}{|c|}{ no measurement } & 1 & \multicolumn{2}{|c|}{ below detection limit } \\
\hline 15008 & $\delta$ Hyi & 4 & $20 \pm 1.4$ & $0.87 \pm 0.08$ & 2 & \multicolumn{2}{|c|}{ below detection limit } & 6 & \multicolumn{2}{|c|}{ below detection limit } \\
\hline 156729 & e Her & - & \multicolumn{2}{|c|}{ no measurement } & 1 & \multicolumn{2}{|c|}{ below detection limit } & 1 & \multicolumn{2}{|c|}{ below detection limit } \\
\hline 168009 & HR 6847 & - & \multicolumn{2}{|c|}{ no measurement } & 1 & \multicolumn{2}{|c|}{ below detection limit } & 1 & \multicolumn{2}{|c|}{ below detection limit } \\
\hline
\end{tabular}

Notes. Model fluxes are listed in Table 1 . The quoted uncertainties of the measurements include the absolute calibration uncertainty of $5 \%$. In the case of only one observation for a specific source, the statistical error of this flux measurement is used in the uncertainty determination, in case of more than one observation for a source, as given in columns $n_{70}, n_{100}$, and $n_{160}$, the standard deviation of the weighted mean from the individual chop/nod fluxes listed in Tables B.1 to B.3, available in electronic form at CDS, is used in the uncertainty calculation. ${ }^{(a)} 1342184583,1342184584$ \& 1342184595 excluded, ${ }^{(b)}$ OBSIDs 1342185441 \& 1342185442 excluded.

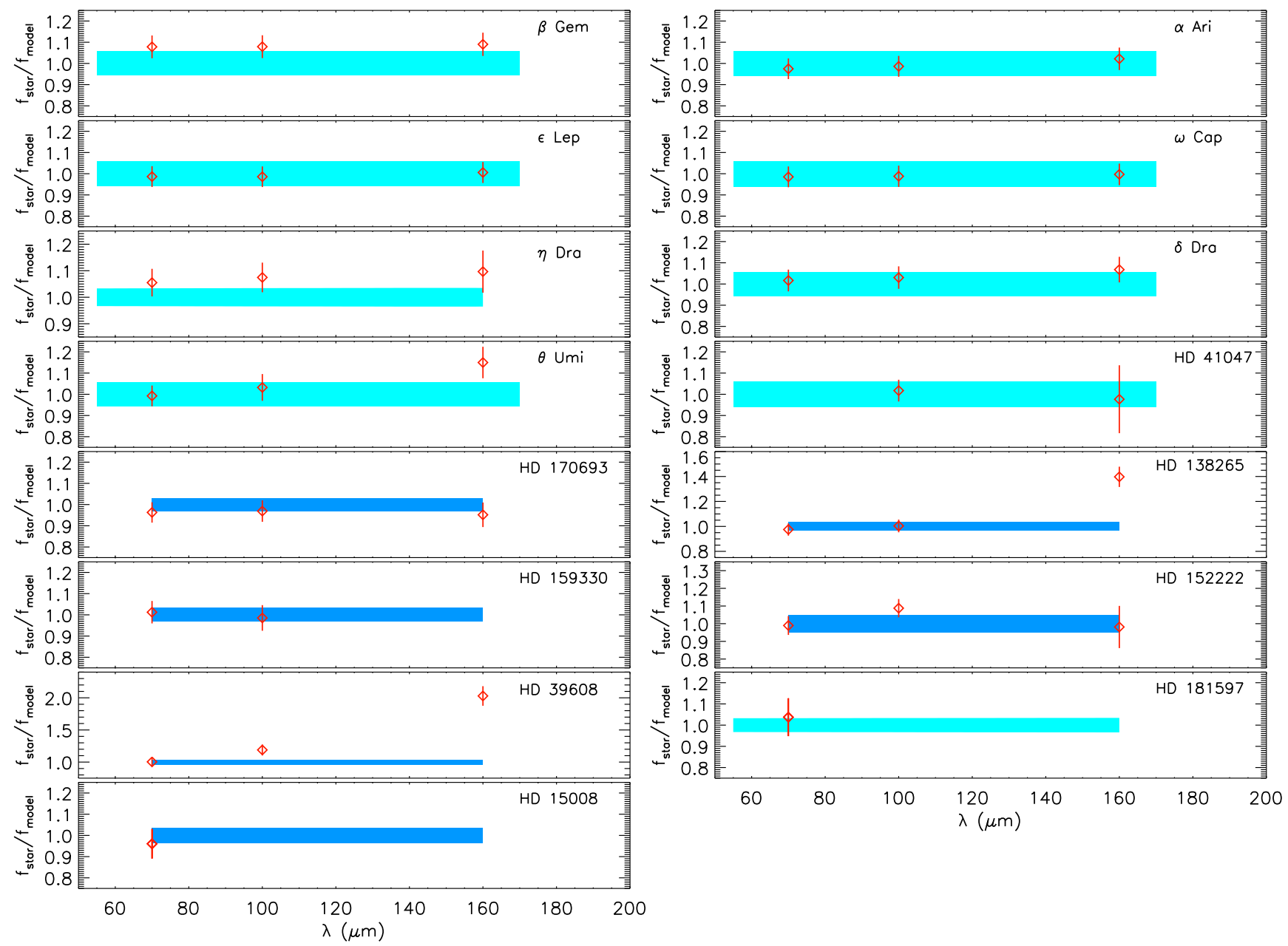

Fig. 7. Ratio of the observed and colour-corrected (cc) scan map photometry fluxes with either the photometric standard model flux or the Gordon et al. (2007) $70 \mu \mathrm{m}$ flux prediction and Rayleigh-Jeans extrapolation (red diamonds). The uncertainty of the models is shown by the light blue range, the uncertainty of the flux prediction and the Rayleigh-Jeans extrapolation by the dark blue range. The uncertainty of the flux ratio includes the absolute photometric error of the measurement. 
Table 9. Evaluation of correspondence of PACS photometry with models or flux predictions $(\mathrm{C}=\mathrm{Cohen}, \mathrm{H}=\mathrm{Hammersley}, \mathrm{G}=\mathrm{Gordon})$.

\begin{tabular}{|c|c|c|c|c|c|c|}
\hline HD & Other name & Model & Mode & Correspondence with model & Reason for deviation & Sect. \\
\hline 62509 & $\beta \mathrm{Gem}$ & $\mathrm{C}$ & $\mathrm{s}, \mathrm{c}$ & no, excess of $\approx+8 \%$ & dust disk in planetary system & 5.2 .1 \\
\hline 12929 & $\alpha$ Ari & $\mathrm{C}$ & $\mathrm{s}$ & yes, better than $\pm 3 \%$ & & \\
\hline 32887 & $\varepsilon$ Lep & $\mathrm{C}$ & $\mathrm{s}, \mathrm{c}$ & yes, better than $\pm 1 \%$ & & \\
\hline 198542 & $\omega$ Cap & $\mathrm{C}$ & $\mathrm{s}$ & yes, better than $\pm 1 \%$ & & \\
\hline 148387 & $\eta$ Dra & $\mathrm{H}$ & $\mathrm{s}, \mathrm{c}$ & no, excess of $\approx+8 \%$ & dust in binary system & 5.2 .1 \\
\hline 180711 & $\delta$ Dra & $\mathrm{C}$ & $\mathrm{s}, \mathrm{c}$ & partially, $\approx+3 \%$ offset for $\lambda \leq 100 \mu \mathrm{m}$ & & \\
\hline & & & & $+7 \% @ 160 \mu \mathrm{m}$ & cirrus confusion & 5.2 .2 \\
\hline 139669 & $\theta$ Umi & $\mathrm{C}$ & $\mathrm{s}, \mathrm{c}$ & $\begin{array}{l}\text { partially, } \approx+3 \% \text { offset for } \lambda \leq 100 \mu \mathrm{m} \\
+16 \% @ 160 \mu \mathrm{m}\end{array}$ & cirrus confusion & 5.2 .2 \\
\hline 41047 & HR 2131 & $\mathrm{C}$ & $\mathrm{s}, \mathrm{c}$ & yes, better than $\pm 3 \%$ & & \\
\hline 170693 & 42 Dra & $\mathrm{G}$ & $\mathrm{s}$ & yes, $\approx-4 \%$ offset & & \\
\hline 138265 & HR 5755 & G & $\mathrm{s}, \mathrm{c}$ & $\begin{array}{l}\text { partially, } \approx-2 \% \text { offset for } \lambda \leq 100 \mu \mathrm{m} \\
+40 \% @ 160 \mu \mathrm{m}\end{array}$ & cirrus confusion & 5.2 .2 \\
\hline 159330 & HR 6540 & G & $\mathrm{s}, \mathrm{c}$ & $\begin{array}{l}\text { partially, better than } \pm 1 \% \text { for } \lambda \leq 100 \mu \mathrm{m} \\
\text { no flux determination } @ 160 \mu \mathrm{m}\end{array}$ & source confusion & 3.7 \\
\hline 152222 & SAO 17226 & $\mathrm{G}$ & $\mathrm{s}, \mathrm{c}$ & yes, better than $\pm 5 \%$ & & \\
\hline 39608 & SAO 249364 & G & $\mathrm{s}$ & $\begin{array}{l}\text { yes, better than 1\%@70 } \mu \mathrm{m} \\
+19 \% @ 100 \mu \mathrm{m},+105 \% @ 160 \mu \mathrm{m}\end{array}$ & background confusion & 5.2 .2 \\
\hline 181597 & HR 7341 & $\mathrm{H}$ & $\mathrm{s}, \mathrm{c}$ & $\begin{array}{l}\text { yes, better than 4\%@70 } \mu \mathrm{m} \\
\text { no flux determination @ } 160 \mu \mathrm{m}\end{array}$ & below detection limit & 3.6 \\
\hline 15008 & $\delta$ Hyi & G & $\mathrm{s}, \mathrm{c}$ & $\begin{array}{l}\text { yes, better than 4\%@70 } \mu \mathrm{m} \\
\text { no flux determination @ } 160 \mu \mathrm{m}\end{array}$ & below detection limit & 3.6 \\
\hline 156729 & e Her & $\mathrm{H}$ & $\mathrm{c}$ & no & below detection limit & \\
\hline 168009 & HR 6847 & $\mathrm{H}$ & $\mathrm{c}$ & no & below detection limit & \\
\hline
\end{tabular}

Notes. Column "mode" specifies the PACS observing mode ( $\mathrm{s}=$ scan map, $\mathrm{c}=$ chop/nod). Column "Reason for deviation" gives a short summary of the discussion in the reference sections.

include the absolute calibration uncertainty of $5 \%$, due to the fiducial star model uncertainties, which is quadratically added to the the rms of the mean flux value.

For six stars we obtain reliable photometry in all three PACS bands. There is no $70 \mu \mathrm{m}$ flux for HD 41047, since there are only measurements in the 100 and $160 \mu \mathrm{m}$ filters. There is no $160 \mu \mathrm{m}$ detection for HD 159330 because of confusion noise. For HD 15008 we only obtain a reliable flux at $70 \mu \mathrm{m}$, since at 100 and $160 \mu \mathrm{m}$ the star is too faint for the applied repetition numbers. HD 181597, HD 156729 and HD 168009 have non-detections despite a high repetition factor of 50 . The nondetection is likely due to a not-yet-perfect knowledge of the optimum observing strategy early in the mission (the observations were Astronomical Observation Template (AOT) test cases). Faintest fluxes, for which the photometry has still good quality (accuracy $\leq 15 \%$ ), are approximately $30 \mathrm{mJy}$.

In Appendix $\mathrm{C}$ we conduct a detailed comparison between the chop/nod and scan map stellar photometry. In summary the results are very consistent and confirm each other. A few cases with larger discrepancy are due to only a small number of measurements or low $\mathrm{S} / \mathrm{N}$ in chop/nod mode.

\section{Comparison with model fluxes or flux predictions}

\subsection{Overview of findings}

Since all detected stars are observed in scan map mode and we have more and better $\mathrm{S} / \mathrm{N}$ measurements in this mode, we restrict the following inter-comparison with the models to scan map photometry. For each star a quantitative comparison per filter is given in Table 7. Figure 7 shows a graphical comparison of the measured fluxes with the model and Table 9 provides a summary of the correspondence evaluation.

We find an excellent correspondence with the model or flux prediction over the full PACS wavelength range for $\alpha$ Ari, $\varepsilon$ Lep, $\omega$ Cap, HD 41047, 42 Dra and HD 152222. We find a partial correspondence up to $100 \mu \mathrm{m}$ for $\delta$ Dra, $\theta \mathrm{Umi}$, HD 138265 and HD 159330, while the $160 \mu \mathrm{m}$ flux exceeds the model flux or, as in the latter case, cannot be determined due to confusion by nearby sources. For HD 39608, the $70 \mu \mathrm{m}$ flux still corresponds excellently with the flux prediction, but at 100 and $160 \mu \mathrm{m}$ a noticeable flux excess is discovered. $\beta \mathrm{Gem}$ and $\eta$ Dra both exhibit a significant offset above the model for all wavelengths. For HD 181597 and HD 15008 we can prove a good correspondence at $70 \mu \mathrm{m}$, but have no means to do so at longer wavelengths, since our measurements are not above the detection limit.

We discuss now the origin of the excess emission for $\delta$ Dra, $\theta$ Umi, HD 138265, HD 39608, $\beta$ Gem and $\eta$ Dra.

\subsection{Stars with FIR excess}

A FIR excess can be an intrinsic source property or be caused by confusing background sources, as already addressed in Sect. 3.7. Cohen et al. (2005) and Dehaes et al. (2011) discuss possible chromospheric emission or thermal emission from an ionized wind which gives noticeable excess at sub-mm wavelengths, but may already set in at FIR wavelengths, as intrinsic emission mechanisms. Groenewegen (2012) investigated the phenomenon of infrared excess around red giant branch stars assuming mass loss arising from chromospheric activity.

One other aspect to consider in this context is possible source variability; we investigate this for the case of $\beta \mathrm{Gem}$ : 
The Cohen et al. (1996) FIR model SED is an extension of an absolutely calibrated $1.2-35 \mu \mathrm{m}_{\text {model }}{ }^{5}$ Cohen et al. (1995). In Fig. 8 we show both model parts represented by the orange and red lines. No inconsistency between both parts can be recognised. Besides the PACS photometry we show the colourcorrected IRAS FSC (Moshir et al. 1989) and SPIRE PSC 6 (Schulz et al. 2017) photometry spanning the wavelength range from 12 to $500 \mu \mathrm{m}$. All measured photometry was taken between 1983 and 2009-2013 and is clearly above the model, meaning that variability of the source is an unlikely explanation, since a major part of the photometric input for the Cohen model was obtained in-between (but IRAS 12 and $25 \mu \mathrm{m}$ photometry was not considered).

Another explanation for an intrinsic FIR excess can be a residual dust disk from a stellar- or planetary-system-formation process. One of the first giant stars, for which an infrared excess was reported, is the K3 giant $\delta$ And (Judge et al. 1987). The giant is the brightest star in a triple or quadruple system and is itself a spectroscopic binary with a companion that is most likely a main sequence K-type star (Bottom et al. 2015). Judge et al. (1987) argued that the infrared excess appears to be caused by a detached primordial dust shell around the giant. Plets et al. (1997) concluded for a larger sample of giants with infrared excess, that this phenomenon is most likely related to the Vega phenomenon around first-ascent giants.

\subsubsection{Intrinsic FIR excess}

$\beta \mathrm{Gem}$ is another good candidate for having a residual debris disk, since it is the host star of a confirmed (Hatzes et al. 2006) Jupiter-sized planet (HD 62509 b, $M=2.9 \pm 0.1 \mathrm{M}_{\mathrm{J}}$, $a=1.69 \pm 0.03 \mathrm{AU}^{7}$ ). A rough estimate (assuming a Jupiter diameter and $T=300 \mathrm{~K}$ ) gives a contrast of $>10^{5}$ between star and planet, meaning that the planet cannot account for the FIR excess of $\approx 8 \%$.

The Cohen models of $\beta \mathrm{Gem}$ are based on $T_{\text {eff }} \approx 4850 \mathrm{~K}$ (see file headers of models with reference to Blackwell et al. 1991). The effective temperature $T_{\text {eff }}$ of giant stars are determined in the ultraviolet to near-infrared wavelength range, either from photometric indices (e.g. Lyubimkov \& Poklad 2014), colours and metallicities (e.g. Alonso et al. 1999), or integrated fluxes and interferometric measurements of the stellar diameters (e.g. Dyck et al. 1996). Other references give $T_{\text {eff }}=$ $4850 \mathrm{~K}$ (Gray et al. 2003) and $4946 \pm 18 \mathrm{~K}$ (Jofré et al. 2015) and thus confirm the value used by Cohen. We have scaled a continuum model ${ }^{8}$ of the PACS fiducial standard star $\alpha$ Boo, a K2III star, by calculating the Selby et al. (1988) $K_{\mathrm{n}}$ narrow band photometry ratio $10^{-0.4 \times(-1.14-(-3.07))}=0.169$. The intention here is not to accurately model the $\beta$ Gem FIR photometry, but to demonstrate that the SED of the cooler source with $T_{\text {eff }}=4320 \mathrm{~K}$ provides a better fit. This indicates that there

\footnotetext{
5 http://general-tools. cosmos.esa.int/iso/users/expl_

lib/ISO/wwwcal/isoprep/cohen/composites/

6 http://irsa.ipac. caltech. edu/cgi-bin/Gator/

nph-scan?submit=Select\&projshort=HERSCHEL;

for SPIRE colour correction, see SPIRE Handbook, Table 5.7

http://herschel.esac.esa.int/Docs/SPIRE/spire_

handbook.pdf

7 The Extrasolar Planet Encyclopedia

http://exoplanet.eu/catalog/HD62509_b/

8 This model can be found under

http://archives.esac.esa.int/hsa/legacy/ADP/

StellarModels/
}

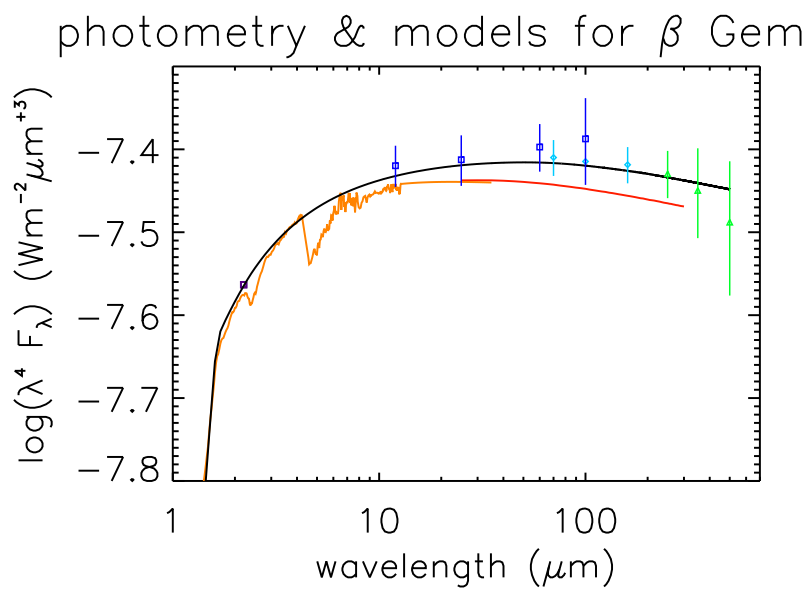

Fig. 8. Investigation of the discrepancy of the $\beta \mathrm{Gem}$ Cohen et al. (1996) model and measured FIR photometry. For a better zoom-in over a large wavelength range, $\log _{10}\left(\lambda^{4} \times f_{\lambda}\right)$ is displayed. The orange and red lines are the Cohen et al. (1995) absolutely calibrated 1.2$35 \mu \mathrm{m}$ spectral model and the Cohen et al. (1996) FIR extension, respectively. The black line represents a scaled $(\times 0.169)$ fiducial star continuum model of $\alpha$ Boo (Dehaes et al. 2011). The scaling factor has been derived from the Selby et al. (1988) $\mathrm{K}_{\mathrm{n}}$ narrow band photometry ratio $10^{-0.4 \times(-1.14+3.07)}$ and the position of the scaling wavelength $(2.205 \mu \mathrm{m})$ is indicated by the violet square symbol. We note that the $\mathrm{K}_{\mathrm{n}}$ zero point is $\approx 3 \%$ higher than that for the Cohen models (cf. file header of $\alpha$ Boo model (reference, see text) vs Table 1 in Cohen et al. 1992). Dark-blue squares, light-blue diamonds and green triangles represent IRAS FSC, PACS and SPIRE photometry and their respective uncertainties. This photometry has been colour-corrected for a $4500 \mathrm{~K}$ blackbody spectrum.

is an additional cooler FIR component beside the photospheric emission of the star. The shape of the SED $>10 \mu \mathrm{m}$ given by the IRAS, PACS, and SPIRE photometry argues for a flat blackbody dust disk (see e.g. Chiang \& Goldreich 1997; Beckwith 1999, for a discussion of the dust disk SED shape depending on its geometry).

$\alpha$ Ari and 42 Dra are also host stars of confirmed Jupiter-sized planets (alf Ari b, $M=1.8 \pm 0.2 \mathrm{M}_{\mathrm{J}}, a=$ 1.2 AU (Lee et al. 2011) ${ }^{9}$; 42 Dra b, $M=3.88 \pm 0.85 \mathrm{M}_{\mathrm{J}}, a=$ 1.19 AU (Döllinger et al. 2009) ${ }^{10}$ ), but for these stars any possible debris disk emission is much fainter than for $\beta \mathrm{Gem}$. The observed SED shape of $\alpha$ Ari is a little bit shallower than the model prediction (cf. Table 7), but the measurement and model uncertainties do not allow any detection. For 42 Dra, no deviation from a pure photospheric emission SED can be found from our photometry. We therefore keep both stars in our standard star list.

In Sect. 3.7 we have shown the $160 \mu \mathrm{m}$ map of $\eta$ Dra (Fig. 6) as a representative example for cirrus confusion. Indeed, from Table 10, $\frac{f_{1006 \text {, corr }}^{10,}}{f_{160, \text { corr }}^{5 !}}=1.17$, which could fully explain $\frac{f_{160}}{f_{\text {model }}}=1.16$ in Table 7 as an excess due to cirrus emission in the standard 10!7-radius aperture. On the other hand, from Fig. 7, we see that already the 70 and $100 \mu \mathrm{m}$ fluxes are off by +6 and $+7 \%$, respectively, with regard to the model. We investigate this discrepancy in Fig. 9 by complementing the PACS photometry with additional IRAS FSC (Moshir et al. 1989)

\footnotetext{
9 The Extrasolar Planet Encyclopedia http://exoplanet.eu/catalog/alfAri_b/

10 http://exoplanet.eu/catalog/42_Dra_b/
} 


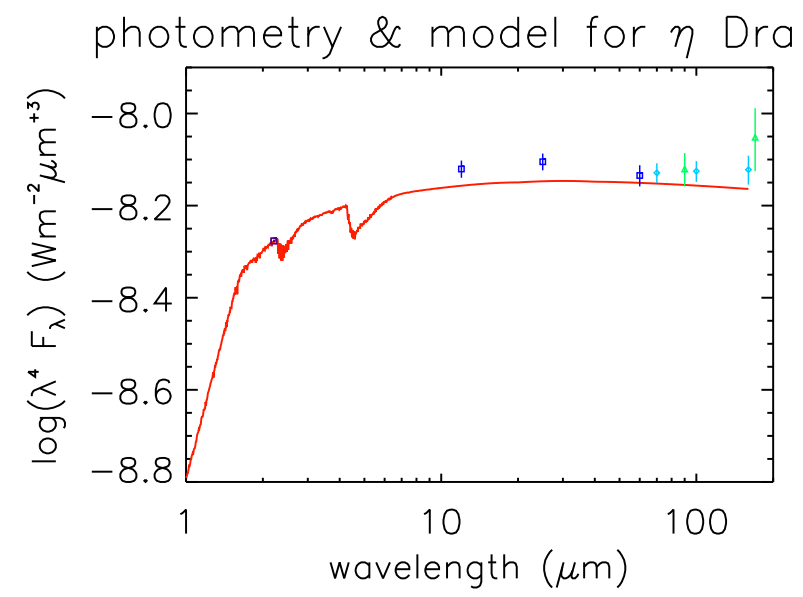

Fig. 9. Investigation of the discrepancy of the $\eta$ Dra Hammersley et al. (1998) model and measured FIR photometry. For a better zoom-in over a large wavelength range, $\log _{10}\left(\lambda^{4} \times f_{\lambda}\right)$ is displayed. The red line is the model, absolutely calibrated at $2.208 \mu \mathrm{m}$ ( $K_{\mathrm{n}}$ magnitude $=0.62 \pm 0.005 \mathrm{mag}$, c.f. Cohen et al. (1999), Selby et al. 1988 ) as represented by the violet square symbol. Dark-blue squares, light-blue diamonds, and green triangles represent IRAS FSC, PACS, and ISOPHOT HPDP photometry and their respective uncertainties. This photometry has been colour-corrected for a $5000 \mathrm{~K}$ blackbody spectrum.

photometry and ISOPHOT (Lemke et al. 1996) Highly Processed Data Products photometry (HPDP, c.f. Appendix. D, Table D.1). It is obvious that all photometric measurements are consistently above the model, irrespective of whether the observations were obtained during the IRAS, IOS, or Herschel missions (1983-2013). The excess is a clear hint of an additional emission component besides the photospheric emission of the star, whereby the rise in flux beyond $100 \mu \mathrm{m}$ is likely caused by cirrus emission. The G8 giant $\eta$ Dra (also identified as CCDM J16239+6130 A) has a K1V companion, CCDM J16239+6130 B (Dommanget \& Nys 2002), at a distance of $2^{\prime \prime} 5$ and at a position angle of $71.6^{\circ} \mathrm{NE}$. The origin of the excess emission could then be dust inside this binary system.

\subsubsection{FIR background confusion}

In Table 10 we have compiled crucial information for those sources whose $160 \mu \mathrm{m}$ fluxes may be contaminated by background confusion. All sources are at relatively high galactic latitudes in the range $23^{\circ} \leq \mid \mathrm{bl} \leq 47^{\circ}$. The $160 \mu \mathrm{m}$ brightness of the ISM, $\mathrm{B}_{160}^{I S M}$, was derived from AKARI-FIS WIDE-L $(140 \mu \mathrm{m})$ all-sky survey maps ${ }^{4}$ (Doi et al. 2015) in the following way:

$B_{160}^{I S M}=\frac{B_{\mathrm{WIDE}-\mathrm{L}}^{\mathrm{AKARI}}-B_{\mathrm{CFIRB}} c c_{\nu^{0}}}{c c_{v^{1.5} \mathrm{BB}(T=20 \mathrm{~K})} K_{v^{1.5} \mathrm{BB}(T=20 \mathrm{~K})}^{F I S 140-P A C S 160}}$,

with $B_{\mathrm{WIDE}-\mathrm{L}}^{\mathrm{AKARI}}$ being the measured AKARI $140 \mu \mathrm{m}$ flux (we highlight that we have transformed the original $6^{\circ} \times 6^{\circ}$ maps in ecliptic coordinates to the equatorial coordinate system and re-centred to the central coordinates of the PACS maps), $B_{\mathrm{CFIRB}}=1.08 \mathrm{MJy} \mathrm{sr}^{-1}$ being the cosmic far-infrared background level (cf. Juvela et al. 2009), $c c_{v^{0}}=0.964$ and $c c_{\gamma^{1.5} \mathrm{BB}(T=20 \mathrm{~K})}=0.95$ are AKARI-FIS WIDE-L colourcorrection factors (Shirahata et al. 2009) for the indexed SEDs and $K_{v^{1.5} \mathrm{BB}(T=20 \mathrm{~K})}^{F I S 140-P A C S 160}=0.954$ is the reference wavelengthcorrection factor between AKARI-FIS WIDE-L and the PACS $160 \mu \mathrm{m}$ filter (Müller et al. 2011) for the modified blackbody SED $v^{1.5} \mathrm{BB}(\mathrm{T}=20 \mathrm{~K})$, which is typical for IR cirrus emission according to latest results (Planck Collaboration XI 2014, Bianchi et al. 2017). The listed surface brightness of $\mathrm{B}_{160}^{I S M}$ is associated with the $15^{\prime \prime} \times 15^{\prime \prime}$ pixel covering the star position, the uncertainty was computed as the standard deviation of the eight neighboring pixel values with regard to the central one. $\mathrm{B}_{160}^{I S M}$ are between 1.3 and $6.0 \mathrm{MJy} \mathrm{sr}^{-1}$, with a gradient with |b|.

Kiss et al. (2005) parameterized the sky confusion noise $(1 \sigma)$ for FIR measurements with ISOPHOT depending on wavelength and background reference configuration geometry as

$$
\frac{N_{\text {conf }}^{\mathrm{PHT}}(\theta, k, \lambda)}{1 \mathrm{mJy}}=C_{0}(\theta, k, \lambda)+C_{1}(\theta, k, \lambda)\left\langle\frac{B(\lambda)-B_{\mathrm{CFIRB}}(\lambda)}{1 \mathrm{MJy} \mathrm{sr}^{-1}}\right\rangle^{\eta(\theta, k, \lambda)} \text {. }
$$

The ISOPHOT C200 measurement configuration $\mathrm{P} / \mathrm{C} / 184^{\prime \prime}$ in Table 4 of Kiss et al. (2005) is closest to our PACS mini-scan-map measurement configuration, except that aperture size and background ring radius have to be scaled down by a factor of $\approx 0.22$ (92" ISOPHOT C200 pixel size vs 19 " PACS "pixel" size corresponding to a circular aperture with 10 .'7 radius and $184^{\prime \prime}$ vs $40^{\prime \prime}$ background ring radius). This means that the PACS sky confusion noise, $N_{\text {conf }}^{\mathrm{PACS}}$, has to be scaled down by a factor of $0.22^{2.5}$ (Kiss et al. 2005) due to the better spatial resolution of PACS. For computation of a point-source representative sky confusion noise we multiply with the aperture correction factor $c_{\text {aper }}(160 \mu m)=1.56$.

$\frac{N_{\text {conf,PS }}^{\mathrm{PACS}}}{1 \mathrm{mJy}}=3.5410^{-2} \times\left[C_{0}+C_{1}\left\langle\frac{B(\lambda)-B_{\mathrm{CFIRB}}(\lambda)}{1 \mathrm{MJy} \mathrm{sr}^{-1}}\right\rangle^{\eta}\right]$.

Applied parameters are $C_{0}=9.3 \pm 6.7 \mathrm{mJy}, C_{1}=3.37 \pm 1.01 \mathrm{mJy}$, and $\eta=1.46 \pm 0.17$.

The $C_{0}$ term represents the confusion noise due to cosmic infrared background fluctuations and amounts to $0.33 \pm 0.24 \mathrm{mJy}$. Berta et al. (2011) give a confusion noise $\sigma_{\mathrm{c}}=0.92 \mathrm{mJy}$ from cosmological fields in the Herschel-PEP survey (obtained for $q=\frac{f_{\text {lim }}}{\sigma_{c}}=5$ ), which corresponds to a $C_{0}$ value equal to $26.0 \mathrm{mJy}$.

The $C_{1}\left\langle B_{160}^{I S M}\right\rangle^{\eta}$ term represents the cirrus confusion noise, which depends on the surface brightness of the emitting cirrus material. With the range $1.3 \leq B_{160}^{I S M} \leq 6.0 \mathrm{MJy} \mathrm{sr}^{-1}$, we predict a cirrus confusion noise $0.18 \pm 0.06 \leq N_{\text {cirrconf,PS }}^{\mathrm{PACS}} \leq$ $1.63 \pm 1.02 \mathrm{mJy}$.

If we attribute the $160 \mu \mathrm{m}$ excess $f_{160 \text {,excess }}$ in Table 10 fully to sky confusion and compare with our confusion noise prediction, we note the following: For our small sample of $160 \mu \mathrm{m}$ excess stars we do not see any dependence on $B_{160}^{I S M}$, in particular for HD 138265 with the lowest $B_{160}^{I S M}=1.27 \mathrm{MJy} \mathrm{sr}^{-1}$, the highest $f_{160 \text {,excess }}=9 \mathrm{mJy}$ is found; and the $1 \sigma$ sky confusion noise derived via Eq. (10) underestimates the actually measured noise by factors 3-25 (1.9-21 accounting for the maximum positive uncertainty).

The confusion noise predictions are average numbers based on a statistical analysis. Peaks and depressions in the sky noise can significantly deviate from the average. The spatial resolution of the AKARI $140 \mu \mathrm{m}$ all-sky survey maps is $\approx 88^{\prime \prime}$ (Takita et al. 2015). The PACS maps reveal much finer structures. Their 
Table 10. Input data for the investigation of $160 \mu \mathrm{m}$ photometric flux contamination of faint stars by background confusion.

\begin{tabular}{|c|c|c|c|c|c|c|c|c|c|}
\hline HD & $\left({ }^{\circ}\right)$ & $\begin{array}{l}\mathrm{b} \\
\left(^{\circ}\right) \\
\end{array}$ & $\begin{array}{c}B_{160}^{I S M} \\
\left(\mathrm{MJy} \mathrm{sr}^{-1}\right) \\
\end{array}$ & OBSIDs & $\begin{array}{c}f_{160, \text { excess }} \\
(\mathrm{mJy})\end{array}$ & $\begin{array}{c}f_{160, \text { corr }}^{5 . !} 35 \\
(\mathrm{mJy})\end{array}$ & $\begin{array}{c}f_{160, \text { corr }}^{10{ }^{\prime}} 7 \\
(\mathrm{mJy})\end{array}$ & $\begin{array}{l}f_{160, \text { corr }}^{14^{\prime \prime} 0} \\
(\mathrm{mJy})\end{array}$ & $\frac{f_{160, \text { corr }}^{10.1}}{f_{160, \text { corr }}^{1 ! 5}}$ \\
\hline 148387 & 92.6 & +41.0 & $2.44 \pm 0.13$ & $1342186146+. .47+. .55+. .56$ & 8.5 & $106.7 \pm 1.4$ & $124.9 \pm 2.1$ & $130.7 \pm 2.6$ & 1.17 \\
\hline 180711 & 98.7 & +23.0 & $6.04 \pm 0.02$ & $1342212497+.498+.499+.500$ & 5.5 & $87.4 \pm 0.9$ & $91.8 \pm 1.1$ & $94.8 \pm 1.2$ & 1.05 \\
\hline- & - & - & - & $1342222147+. .48+. .49+. .50$ & - & $90.8 \pm 1.0$ & $94.4 \pm 1.1$ & $95.3 \pm 1.3$ & 1.04 \\
\hline- & - & - & - & $1342233571+. .72+. .73+. .74$ & - & $92.0 \pm 1.0$ & $95.9 \pm 1.1$ & $99.0 \pm 1.2$ & 1.04 \\
\hline- & - & - & - & $1342250093+. .94+. .95+. .96$ & - & $87.2 \pm 0.9$ & $85.9 \pm 1.0$ & $84.2 \pm 1.1$ & 0.99 \\
\hline- & - & - & - & $1342257987+. .88+. .89+. .90$ & - & $86.2 \pm 0.9$ & $91.4 \pm 1.1$ & $95.2 \pm 1.3$ & 1.06 \\
\hline 139669 & 112.9 & +36.5 & $2.67 \pm 0.07$ & $1342191982+. .83$ & 8.0 & $61.3 \pm 0.7$ & $66.5 \pm 0.9$ & $68.4 \pm 1.0$ & 1.08 \\
\hline 138265 & 95.8 & +47.4 & $1.27 \pm 0.03$ & $1342188841+42$ & 9.0 & $30.1 \pm 0.4$ & $33.3 \pm 0.5$ & $34.9 \pm 0.6$ & 1.11 \\
\hline 39608 & 269.6 & -30.9 & $5.22 \pm 0.17$ & $1342198535+. .36+. .37+. .38$ & 6.0 & $10.7 \pm 0.2$ & $13.5 \pm 0.3$ & $15.9 \pm 0.4$ & 1.26 \\
\hline 152222 & 98.5 & +36.7 & $2.57 \pm 0.15$ & $1342227973+. .74$ & - & $8.2 \pm 0.2$ & $7.0 \pm 0.3$ & $5.9 \pm 0.4$ & 0.85 \\
\hline
\end{tabular}

Notes. The determination of $B_{160}^{I S M}$ is described in the text. The OBSID combinations of the deepest maps are used for this investigation. $f_{160 \text {,excess }}$ is estimated as the difference of the measured $f_{160}$ minus model $f_{\text {model }}$ flux from Table 7 . Listed fluxes $f_{160, \text { corr }}^{\text {apertius }}$ are not colour corrected. Uncertainties of the flux estimates include a $1 \%$ uncertainty of the aperture correction to obtain the total flux, which is quadratically added to $\sigma$ aper (c.f. Eq. (2)).

weight to the noise is much higher than to the average surface brightness. Therefore, calculating the cirrus noise from the surface brightness of a larger area will always underestimate the local cirrus noise. Another possibility is that the PSF of a discrete few-mJy source coincides - accidentally to a large percentage - with the PSF of the star. Differential number counts in cosmological fields, as in Fig. 7 of Berta et al. (2011), suggest that there are $9.2 \times 10^{3}$ background sources/deg ${ }^{2}$ for $f_{\text {lim }} \geq 3.5 \mathrm{mJy}$, which gives 0.25 sources per photometric aperture of $10 ! 7$, hence an already high likelihood that such a source can blend the photometry of our faint stars. We cannot exclude either that in some maps some amount of the $160 \mu \mathrm{m}$ excess is produced by the data reduction scheme itself by reducing the background level in some of the background reference areas (this can vary from map to map depending on the actual detector drift behaviour along the time-line and the level of reduction).

To some extent a contribution by an underlying source can be disentangled via multi-aperture photometry which includes aperture sizes as small as the PSF FWHM. From multiaperture photometry of the deepest maps (OBSID combinations are indicated in Table 10) with aperture radii 5'.35, 10.7 (the default one for our photometric analysis), and 14.'0, we see that the flux increase is usually greater than the associated uncertainties, which is a hint of flux contribution by another source. As a reference, we also include the multiaperture photometry of HD 152222 which does not show any flux increase (rather a flux decrease due to increasing uncertainties in the background subtraction with larger aperture size).

In Fig. 10 we investigate the nature of the $160 \mu \mathrm{m}$ sky background structure, both on an absolute level and larger scale with the help of the AKARI-FIS WIDE-L $(140 \mu \mathrm{m})$ all-sky survey maps ${ }^{4}$ (Doi et al. 2015) and on the PACS scale by parallel JScanam processing of the maps which tends to preserve more reliably small-scale structured extended emission, while the larger-scale background is subtracted. In the following, we discuss the sources $\delta$ Dra, $\theta$ Umi, HD 138265, HD 39608 and HD 152222 individually with regard to level and nature of their background confusion.
For $\delta$ Dra the scan map photometry in Table 10 gives on aver-

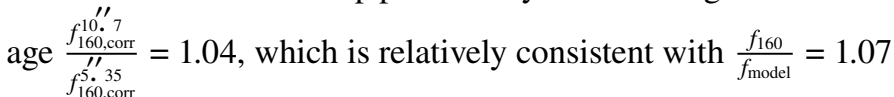
in Table 7, given that also the 70 and $100 \mu$ m fluxes are $2-3 \%$ above the model. The AKARI-map in Fig. 10 shows that the source is located at the wing of a cirrus knot. The JScanam map reveals filamentary structure around the source, which indicates that the small excess in the order of $4 \%$ is likely by IR cirrus contamination. One out of the five cases investigated in Table 10 does not indicate any excess and the measured 10! 7 aperture flux is close to the model flux. This is an example of the ability of the scan map orientation to influence the structure of the source environment due to the HPF running along the scan direction.

For $\theta$ Umi the scan map photometry gives $\frac{f_{160,7}^{10,7}}{f_{160, \text {, corr }}^{\prime \prime \prime}, 35}=1.08$, which is relatively consistent with $\frac{f_{160}}{f_{\text {model }}}=1.13$ in Table 7, meaning that the $160 \mu \mathrm{m}$ photometry in the small aperture is quite close to the model flux. The AKARI-map in Fig. 10 shows that the source is located on a cirrus filament. The JScanam map reveals filamentary structure, too, coinciding in direction with the AKARI-map feature, which supports that the excess found for the default photometric aperture of $10 .^{\prime} 7$ is fully accounted for by IR cirrus contamination.

HD 138265 exhibits a noticeable FIR excess at $160 \mu \mathrm{m}$ only, which makes it a potential background-contaminated source, too. The AKARI-map in Fig. 10 shows that it is located on the wing of a small faint cirrus knot. The JScanam processed map indicates filamentary knotty structure mostly east, south, and west of the source, which fits to its location on the knot. The morphology of the filamentary structure resembles cirrus emission rather than compact sources. We derive $\frac{f_{100, \text { corr }}^{10^{\prime \prime} 7}}{f_{160, \text { corr }}^{5 !}}=1.11$, which only partially explains $\frac{f_{160}}{f_{\text {model }}}=1.36$. In this case the background contribution may be more centered in the beam, meaning that it cannot be fully separated by the multi-aperture method.

HD 39608 shows the by far strongest $160 \mu \mathrm{m}$ excess, with $\frac{f_{160}}{f_{\text {model }}}=2.20$. Our multi-aperture photometry gives only 

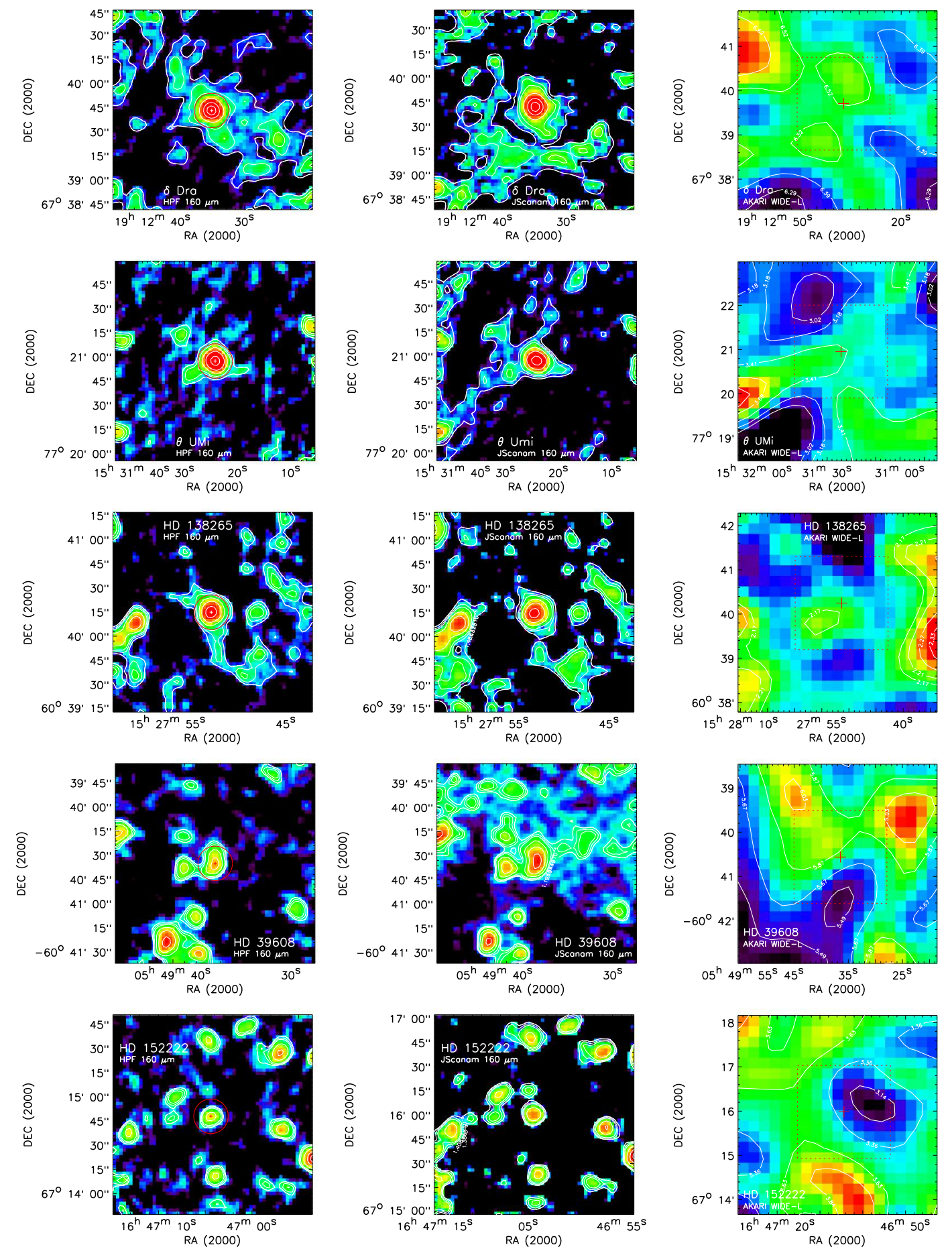

Fig. 10. Investigation of the $160 \mu \mathrm{m}$ sky background structure around the sources $\delta$ Dra (OD 934), $\theta$ UMi (OD 160), HD 138265 (OD 233 ), HD 39608 (OD 400) and HD 152222 (OD 843) from top to bottom. The deepest available maps were used, see Table 10 for the OBSID combination and Table A.5, available in electronic form at CDS, for the observation details. The left panel shows the HPF processed map used for the photometry, the photometric aperture with 10!7 radius is indicated by the red circle and a small red or white cross in its centre. The middle panel shows the JScanam processed map which should reproduce extended emission more reliably. The JScanam map of $\delta$ Dra shows the superposition of all five sets of OBSIDs in Table 10 (ODs 607, 751, 934, 1198, and 1328) as the deepest image of this field. The right panel shows the AKARI WIDE-L $(140 \mu \mathrm{m})$ background emission around the source (red cross), the AKARI map area is about four times as large as the PACS map area, which is indicated by the red dashed square.

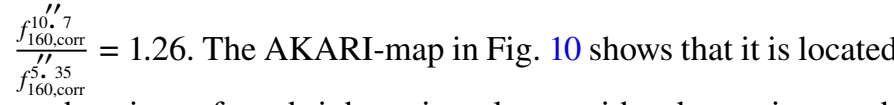
on the wings of two brighter cirrus knots with a depression south of it. The JScanam map reveals that there is extended filamentary structure mainly north of the source, in agreement with the larger-scale feature of the AKARI-map. This also affects the area where the background is determined. Given that the source is one of the faintest in our sample, with an expected photospheric flux of only about $6 \mathrm{mJy}$, any inaccuracy in the background determination has a severe impact on the resulting source flux. Furthermore, the source looks elongated in the north-south direction, which indicates contaminating emission 
Table 11. $K$-band and PACS $100 \mu \mathrm{m}$ photometry and a selection of stellar parameter information for the PACS fiducial primary standards (status: "f") and PACS faint star primary standard candidates (status: "c") with nearly identical spectral type.

\begin{tabular}{lccccccccc}
\hline \hline Name & Status & SpType & $\begin{array}{c}V \\
(\mathrm{mag})\end{array}$ & $\begin{array}{c}K \\
(\mathrm{mag})\end{array}$ & $\begin{array}{c}K_{\mathrm{n}}^{p 3} \\
(\mathrm{mag})\end{array}$ & $\begin{array}{c}K_{\mathrm{s}, 2 \mathrm{MASs}}^{p 4} \\
(\mathrm{mag})\end{array}$ & $\begin{array}{c}f_{100}^{p 5} \\
(\mathrm{mJy})\end{array}$ & $\begin{array}{c}T_{\mathrm{eff}} \\
(\mathrm{K})\end{array}$ & $\begin{array}{c}\Theta_{\mathrm{d}} \\
\mathrm{m}^{\prime \prime}\end{array}$ \\
\hline$\alpha$ Boo & $\mathrm{f}$ & K2III & -0.04 & - & $-3.07 \pm 0.01$ & - & $7509 \pm 375$ & $4320 \pm 140^{s 1}$ & $20.74 \pm 0.10^{s 1}$ \\
$\alpha$ Ari & $\mathrm{c}$ & K1IIIb & 2.01 & $-0.63 \pm 0.03^{p 1}$ & - & - & $820 \pm 41$ & $4636 \pm 13^{s 2}$ & $6.90 \pm 0.07^{s 5}$ \\
42 Dra & $\mathrm{c}$ & K1.5III & 4.82 & $1.95 \pm 0.05^{p 2}$ & - & - & $73.1 \pm 3.8$ & $4446 \pm 12^{s 2}$ & $2.03 \pm 0.03^{s 5}$ \\
HD 159330 & $\mathrm{c}$ & K2III & 6.21 & - & - & $2.787 \pm 0.206$ & $31.0 \pm 1.9$ & - & - \\
HD 152222 & $\mathrm{c}$ & K2III & 7.03 & - & - & $3.654 \pm 0.238$ & $20.8 \pm 1.0$ & - & - \\
$\alpha$ Tau & $\mathrm{f}$ & K5III & 0.85 & - & $-2.94 \pm 0.01$ & - & $6909 \pm 345$ & $3850 \pm 140^{s 1}$ & $20.89 \pm 0.10^{s 1}$ \\
$\gamma$ Dra & $\mathrm{f}$ & K5III & 2.23 & - & $-1.38 \pm 0.01$ & - & $1604 \pm 80$ & $3960 \pm 140^{s 1}$ & $9.94 \pm 0.05^{s 1}$ \\
$\varepsilon$ Lep & $\mathrm{c}$ & K4III & 3.18 & $-0.20 \pm 0.03^{p 1}$ & - & - & $568 \pm 28$ & $4243 \pm 25^{s 2}$ & $6.08 \pm 0.06^{s 5}$ \\
HD 41047 & $\mathrm{c}$ & K5III & 5.52 & - & - & $1.740 \pm 0.218$ & $96.7 \pm 4.9$ & - & $2.47 \pm 0.03^{s 4}$ \\
$\theta$ Umi & $\mathrm{c}$ & K5III- & 4.98 & $1.33 \pm 0.05^{p 2}$ & - & - & $144 \pm 8.8$ & - & $2.97 \pm 0.04^{s 5}$ \\
HD 138265 & $\mathrm{c}$ & K5III & 5.90 & $2.38 \pm 0.04^{p 2}$ & - & - & $56.5 \pm 2.9$ & $3758 \pm 166^{s 3}$ & $2.06 \pm 0.044^{s 3}$ \\
$\beta$ And & f & M0III & 2.06 & - & $-1.93 \pm 0.01$ & - & $2737 \pm 137$ & $3880 \pm 140^{s 1}$ & $13.03 \pm 0.06^{s 1}$ \\
$\omega$ Cap & $\mathrm{c}$ & M0III & 4.12 & $0.21 \pm 0.03^{p 1}$ & - & - & $413 \pm 21$ & $3760 \pm 150^{s 4}$ & $5.16 \pm 0.06^{s 5}$ \\
\hline
\end{tabular}

Notes. Fiducial primary standards and related candidates are grouped together. $V$-band photometry is given for completeness and for the conversion of the $K$ magnitude between the (Selby et al. 1988) $K_{\mathrm{n}}$ narrow band photometric system and the Johnson $K$-band photometric system. ${ }^{1}$ Star is a proven reliable standard only up to $100 \mu \mathrm{m}$ due to background confusion. ${ }^{\left({ }^{1}\right)}$ Ducati $(2002$, catalogue of stellar photometry in Johnson's 11 -colour system). ${ }^{(p 2)}$ Neugebauer \& Leighton $\left(1969\right.$, two-micron sky survey). ${ }^{(p 3)}$ Selby et al. (1988). ${ }^{\left({ }^{4}\right)}$ Cutri et al. (2003, 2MASS all-sky catalogue of point sources) Note: For $K_{\mathrm{s}, 2 \mathrm{MASs}}<4$ mag photometric uncertainties are high, because fluxes were estimated from a fit to the wings of the saturated stellar profile (Skrutskie et al. 2006). ${ }^{(p 5)}$ For the fiducial standards we use the continuum model ${ }^{8}$ flux with an uncertainty of $5 \%$; for the candidate stars we use the flux from scan map photometry. ${ }^{(s 1)}$ Dehaes et al. (2011). ${ }^{(s 2)}$ Jofré et al. (2015). ${ }^{\left({ }^{3}\right)}$ Baines et al. $(2010) .{ }^{\left({ }^{(s)}\right)}$ Tsuji (1981). ${ }^{(s 5)}$ Cohen et al. (1999).

inside the measurement aperture. From Fig. 7, we see that there is already a noticeable excess of $19 \%$ at $100 \mu \mathrm{m}$. Unfortunately we cannot investigate this properly on a JScanam processed map, since there exists only one map in one scan direction (the cross-scan map was erroneously executed in the $70 \mu \mathrm{m}$ filter). HD 39608 has the second strongest ISM sky background $B_{160}^{I S M}$ (c.f. Table 10), meaning that it is very likely that already at $100 \mu \mathrm{m}$ there can be significant sky background contamination. The deep combined $70 \mu \mathrm{m}$ map shows an elongated emission feature underneath the source, too.

For comparison we also show the environment of HD 152222 in Fig. 10, which is only slightly brighter than HD 39608. The AKARI-map shows that it is located outside a cirrus ridge close to a depression in the cirrus emission. The JScanam map reveals that the area around it is also crowded, but the sources are discrete compact sources, which argues for an extragalactic nature, and besides the star itself appears isolated inside the measurement aperture. The derived flux is quite consistent with the model flux which argues against a systematic background underestimate in this source flux range.

HD 39608 is hence no longer qualified as a potential calibration standard. $\theta$ Umi and HD 138265 can be considered as suitable standards up to $100 \mu \mathrm{m}$.

\section{Establishment of new faint FIR primary standards}

Primary flux standards are used for absolute flux calibration. Their SED is assumed to be known and stable or predictable. Absolute calibration of these sources is achieved either by a direct method, like comparison against a blackbody source, or by a indirect method, for example stellar or planetary atmosphere models. Deustua et al. (2013) give a detailed description of absolute calibration of astronomical flux standards. Primary flux standards in the far-infrared wavelength range are, with decreasing brightness, planets (Müller et al. 2016), asteroids (Müller et al. 2014), and stars (Dehaes et al. 2011), which are all calibrated via the indirect method and verified by independently calibrated multi-wavelength flux measurements. The best achievable uncertainties are currently 5-7\%.

The Cohen et al. (1996) models of $\alpha$ Ari, $\varepsilon$ Lep, $\omega$ Cap, $\delta$ Dra and HD 41047 are well confirmed by our PACS photometry and are thus adequate representations of the stellar FIR photospheric emission. These stars together with 42 Dra and HD 152222 are good candidates to establish fainter FIR primary standards. This list is complemented by $\theta$ Umi, HD 159330, and HD 138265 for which we can confirm a reliable FIR spectrum only up to $100 \mu \mathrm{m}$ due to neighbouring source- or cirrus confusion at longer wavelengths.

As already discussed earlier, the Cohen et al. (1996) models are FIR extensions of absolutely calibrated $1.2-35 \mu \mathrm{m}$ template spectra (Cohen et al. 1995, 1999). Another set of models ranging from $0.7 \mu \mathrm{m}$ to $7 \mathrm{~cm}$ was developed by Dehaes et al. (2011) for the Herschel-PACS fiducial primary standards. Several of our faint primary standard candidates have the same or similar spectral type as one of the PACS primary standard stars. As a first model approximation we can scale these fiducial standard star models to the flux levels of our primary standard candidates. For an accurate model one would have to run a flux model code taking into account the stellar parameter information of each star, a project which is beyond the scope of this paper.

$\delta$ Dra with spectral type G9III has no suitable counterpart among the Herschel-PACS fiducial primary standards, since the earliest spectral type is K2III. But we note that it was modelled earlier by Decin et al. (2003) as IRAS-SWS calibrator. We do not include $\delta$ Dra in Table 11, but refer to Table 3 in Decin et al. (2003) which gives its stellar properties. 
Table 12. Determination of the scale factor to adjust the related fiducial primary standard star model to the flux level of the candidate primary standard.

\begin{tabular}{llccccc}
\hline \hline $\begin{array}{l}\text { Primary } \\
\text { candidate }\end{array}$ & $\begin{array}{l}\text { Primary } \\
\text { standard }\end{array}$ & Kmag ratio & $\mathrm{f}_{100}$ ratio & Scale factor & \% Scale uncert. & \% Abs. uncert. \\
\hline$\alpha$ Ari & $\alpha$ Boo & $1.11 \pm 0.04 \times 10^{-1}$ & $1.09 \pm 0.11 \times 10^{-1}$ & $1.11 \pm 0.01 \times 10^{-1}$ & 0.9 & 5.2 \\
42 Dra & $\alpha$ Boo & $1.03 \pm 0.06 \times 10^{-2}$ & $9.73 \pm 1.00 \times 10^{-3}$ & $1.01 \pm 0.03 \times 10^{-2}$ & 3.0 & 5.9 \\
HD 152222 & $\alpha$ Boo & $2.07 \pm 0.46 \times 10^{-3}$ & $2.77 \pm 0.27 \times 10^{-3}$ & $2.59 \pm 0.31 \times 10^{-3}$ & 12.0 & 13.0 \\
HD 159330 & $\alpha$ Boo & $4.58 \pm 0.92 \times 10^{-3}$ & $4.13 \pm 0.46 \times 10^{-3}$ & $4.22 \pm 0.18 \times 10^{-3}$ & 4.3 & 6.7 \\
$\varepsilon$ Lep & $\alpha$ Tau & $8.49 \pm 0.31 \times 10^{-2}$ & $8.22 \pm 0.82 \times 10^{-2}$ & $8.46 \pm 0.09 \times 10^{-2}$ & 1.1 & 5.2 \\
$\varepsilon$ Lep & $\gamma$ Dra & $3.56 \pm 0.13 \times 10^{-1}$ & $3.54 \pm 0.35 \times 10^{-1}$ & $3.56 \pm 0.007 \times 10^{-1}$ & 0.2 & 5.1 \\
HD 41047 & $\alpha$ Tau & $1.37 \pm 0.29 \times 10^{-2}$ & $1.40 \pm 0.14 \times 10^{-2}$ & $1.39 \pm 0.01 \times 10^{-2}$ & 0.7 & 5.1 \\
HD 41047 & $\gamma$ Dra & $5.73 \pm 1.21 \times 10^{-2}$ & $6.03 \pm 0.61 \times 10^{-2}$ & $5.97 \pm 0.12 \times 10^{-2}$ & 2.0 & 5.5 \\
$\theta$ Umi $^{1}$ & $\alpha$ Tau & $2.07 \pm 0.12 \times 10^{-2}$ & $2.08 \pm 0.23 \times 10^{-2}$ & $2.07 \pm 0.004 \times 10^{-2}$ & 0.2 & 5.1 \\
$\theta$ Umi $^{1}$ & $\gamma$ Dra & $8.71 \pm 0.48 \times 10^{-2}$ & $8.98 \pm 1.00 \times 10^{-2}$ & $8.76 \pm 0.11 \times 10^{-2}$ & 1.3 & 5.3 \\
HD 138265 & $\alpha$ Tau & $7.88 \pm 0.36 \times 10^{-3}$ & $8.18 \pm 0.83 \times 10^{-3}$ & $7.93 \pm 0.11 \times 10^{-3}$ & 1.4 & 5.3 \\
HD 138265 & $\gamma$ Dra & $3.31 \pm 0.15 \times 10^{-2}$ & $3.52 \pm 0.36 \times 10^{-2}$ & $3.34 \pm 0.07 \times 10^{-2}$ & 2.2 & 0.7 \\
$\omega$ Cap & $\beta$ And & $1.48 \pm 0.06 \times 10^{-1}$ & $1.51 \pm 0.15 \times 10^{-1}$ & $1.48 \pm 0.01 \times 10^{-1}$ & 5.6 & 5.1 \\
\hline
\end{tabular}

Notes. The last two columns list the uncertainty percentage of the scale factor and the absolute uncertainty of the scaled model approximation, the latter being the Gaussian error propagation of the scale uncertainty, $1 \%$ uncertainty in the stellar parameters and 5\% uncertainty of the models. ${ }^{(1)}$ Star is a proven reliable standard only up to $100 \mu \mathrm{m}$ due to background confusion.
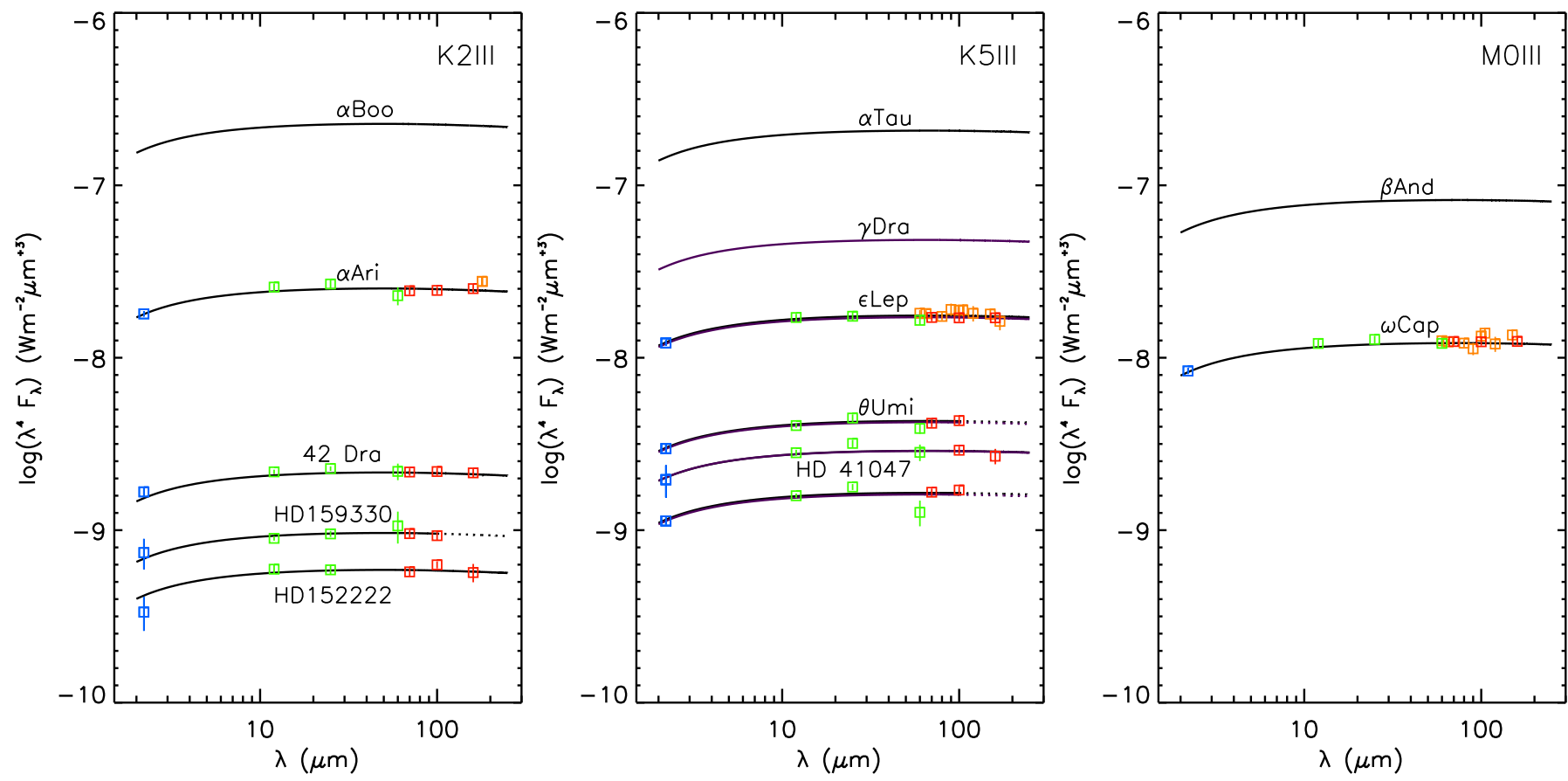

Fig. 11. Scaling of PACS fiducial star continuum models (black and purple lines) to the flux level of the primary standard candidates applying the scale factors of Table 12. For a better zoom-in over a large wavelength range, $\log _{10}\left(\lambda^{4} \times f_{\lambda}\right)$ is displayed. Blue squares are the $K$-band photometry, green squares are colour-corrected IRAS FSC photometry (Moshir et al. 1989), orange squares are ISOPHOT HPDP photometry (Lemke et al. 1996, see Appendix D) and red squares are PACS photometry. In the middle panel the scaled models of both $\alpha$ Tau (black) and $\gamma$ Dra (purple) are plotted. Dashed parts of the SEDs of HD 159330, $\theta$ Umi, and HD 138265 indicate that these stars are proven reliable standards only up to $100 \mu \mathrm{m}$ due to background confusion.

In Table 11 we have compiled photometry and stellar properties of the fiducial primary standards and our primary standard candidates which match in spectral type. Jofré et al. (2015) provide essential stellar parameters for $\alpha$ Ari, $\varepsilon$ Lep, and 42 Dra, therefore a dedicated flux model code could be run.

In Table 12 we compile the $K$-magnitude ratio and the $100 \mu \mathrm{m}$ flux ratio for matching pairs of fiducial primary standards and primary standard candidates. From both ratios we compute the scale factor for the fiducial star model as a weighted mean. We apply the following transformations between the different $K$-band photometric systems: (1) $V-K=-0.020+$ $0.989 \times\left(V-K_{\mathrm{n}}\right)$ (Selby et al. 1988) and (2) $K=K_{\mathrm{s}, 2 \mathrm{MASS}}+$ 0.044 (Bessel 2005). Scale factors are between 0.356 and 0.0026 , that is, the faintest primary standard candidate, HD 152222, is about 360 times fainter than the related fiducial primary standard $\alpha$ Boo and still about 80 times fainter than the faintest fiducial primary standard, $\gamma$ Dra. Table 12 also gives the percentage of the uncertainty of the scaling. We estimate the uncertainty due to 
variation in stellar parameters, such as the effective temperature, by scaling the fiducial model of $\alpha$ Tau to the level of $\gamma$ Dra, which are both K5III stars. The difference over the wavelength range $2-250 \mu \mathrm{m}$ is less than $0.8 \%$. We therefore adopt an uncertainty of $1 \%$ due to variations in stellar parameters. Given, that the fiducial primary standards have an absolute accuracy of 5\%, then the absolute uncertainty of the scaled model approximation, as listed in the last column of Table 12, can be determined as the sum of the three uncertainty terms described above.

Figure 11 shows the scaling of the fiducial standard star models to the flux levels of our primary standard candidates and a verification with available photometry. $K$-band and PACS photometry are supplemented by IRAS FSC photometry (Moshir et al. 1989) and in some cases by ISOPHOT HDPD photometry (Lemke et al. 1996, see Appendix D).

For $\alpha$ Ari, $\varepsilon$ Lep, $\omega$ Cap, HD 41047 and 42 Dra the derived absolute photometric uncertainty is in the range 5-6\%, hence they are well suited new FIR primary standards, which are about 2-20 times fainter than our faintest fiducial primary standard $\gamma$ Dra. Only for the faintest star, HD 152222, does the higher uncertainty of the scaling factor result in a derived absolute uncertainty of $13 \%$. A major driver for this high scaling uncertainty is the high uncertainty of the publicly available $K$-band photometry (cf. Table 11 , footnote $\mathrm{p} 4$ ), meaning that the K-magnitude ratio and the $100 \mu \mathrm{m}$ flux ratio do not match well. From Fig. 11 it is obvious that a more accurate $K$-band photometry would certainly help to bring this star into a similar absolute photometric uncertainty range to the brighter ones.

For $\theta$ Umi, HD 138265 and HD 159330, which are substantially affected by neighbouring source- or cirrus confusion at $160 \mu \mathrm{m}$ (Sects. 3.7 and 5.2), clean photometry can be obtained up to $100 \mu \mathrm{m}$ with a telescope of angular resolution similar to Herschel, which leads to an equally good absolute photometric uncertainty in the range 5-7\%. Also, for HD 159330, improved $K$-band photometry can further reduce its resulting absolute photometric uncertainty. We therefore keep these three sources as reliable standards up to $100 \mu \mathrm{m}$, but with the strong caveat not to use them beyond this wavelength. Only with a considerably higher angular resolution than Herschel could the confusion issues of these sources be overcome at $160 \mu \mathrm{m}$.

HD 138265, HD 159330, and HD 152222 will be observable with the James Webb Space Telescope MIRI Imager at $20 \mu \mathrm{m}$ in bright source mode with the $64 \times 64$ sub-array (Bouchet et al. 2015).

\section{Conclusions}

The PACS faint star sample with 14 giant and 3 dwarf stars has allowed a comprehensive sensitivity assessment of the PACS photometer and provided accurate photometry for detailed SED investigation and establishment of a set of faint FIR primary standard candidates for use by future space missions.

For PACS scan maps, the recommended scientific observation mode for the PACS photometer, we have described a consistent method for how to derive $\mathrm{S} / \mathrm{Ns}$, based on a robust noise measurement with the help of a flux histogram restricted to the applicable map coverage value range. The comparison with the $\mathrm{S} / \mathrm{N}$ predictions of the exposure time calculation tool in the Herschel Observatory Planning Tool HSpot has resulted in very good consistency, proving the tools for PACS photometry observation planning as very reliable. We have demonstrated that the underlying assumptions of the tool, that the $\mathrm{S} / \mathrm{N}$ scales linearly with flux and with the square root of the observing time, are valid over large ranges of flux and time. A restriction appears for the $160 \mu \mathrm{m}$ filter, where source confusion often limits the gain in $\mathrm{S} / \mathrm{N}$ with increasing observing time. We could also show that scan maps obtained with the recommended scan speed of $20^{\prime \prime} / \mathrm{s}$ yield a higher $\mathrm{S} / \mathrm{N}$ than scan maps with $10^{\prime \prime} / \mathrm{s}$, the scan speed favoured pre-flight.

We have shown that in the case of faint sources, small aperture sizes (with a radius of the size of the PSF FWHM) reduces background noise inside the aperture and optimizes the accuracy of the flux determination.

We have obtained reliable photometry for 11 stars in all three PACS filters (at 70, 100, and $160 \mu \mathrm{m}$ ). For one further star we have obtained reliable 100 and $160 \mu \mathrm{m}$ photometry. For one more star we have obtained reliable 70 and $100 \mu \mathrm{m}$ photometry only, $160 \mu \mathrm{m}$ photometry being limited here by confusion of neighbouring sources. For two other stars we have obtained reliable photometry only at $70 \mu \mathrm{m}$, a detection at longer wavelengths being limited by sensitivity limitations and confusion noise. The two faintest sources observed in chop/nod mode have not been detected at all despite high repetition factors of the basic chop/nod pattern. The non-detection is likely due to a not-yet-perfect knowledge of the optimum observing strategy early in the mission. Faintest fluxes, for which the photometry has still good quality, are about $10-20 \mathrm{mJy}$ for the scan map observations and $30 \mathrm{mJy}$ for the available chop/nod observations.

For the faintest star at $160 \mu \mathrm{m}$ with reliable photometry in all three filters, HD 152222, we have conducted an inter-comparison of the HPF photometry from the deepest map with the results of three additional Herschel mapper softwares, namely JScanam, Scanamorphos and Unimap. All four mappers allow us to obtain sound photometry in all three filters. We have identified the level of qualitative consistency as well as some systematic differences with regard to photometry, noise, and beam profiles among the four mappers. A more systematic and quantitative photometric performance comparison of the four mappers will be the subject of a dedicated publication.

For the 12 stars with reliable photometry out to $160 \mu \mathrm{m}$ we can prove that 7 stars are consistent with models or flux predictions for pure photospheric emission. $\delta$ Dra has a slight $160 \mu \mathrm{m}$ excess due to cirrus contamination of the order of $4 \%$, but this is still within the overall uncertainty margin. Two stars show excess emission over the whole $(>10 \mu \mathrm{m})$ FIR range. For $\beta \mathrm{Gem}$ (Pollux), which is the host star of a confirmed Jupiter-sized exoplanet, we conclude from our photometry results that it has in addition a flat blackbody dust disk. The G8 giant $\eta$ Dra has a K1 dwarf companion, therefore the origin of the excess emission likely arises from dust inside this binary system. For three stars with $160 \mu \mathrm{m}$ fluxes below $60 \mathrm{mJy}$ we find $160 \mu \mathrm{m}$ excesses in the order 6 to $9 \mathrm{mJy}$. Investigation of the $160 \mu \mathrm{m}$ absolute sky brightness with the help of AKARI-maps, the filamentary emission structure in the environment of the source on the PACS maps, and multi-aperture PACS photometry strongly support an explanation of this excess as being due to sky background confusion. This is a combination of cirrus confusion affecting the background subtraction and faint underlying objects inside the photometric aperture around the star affecting the source profile. The faintest star at $70 \mu \mathrm{m}$ with reliable photometry in all three filters, HD 39608, is heavily affected by sky confusion noise from $100 \mu \mathrm{m}$ onwards and has therefore to be excluded as a primary standard candidate.

The seven stars with pure photospheric emission over the full PACS wavelength range, $\alpha$ Ari, $\varepsilon$ Lep, $\omega$ Cap, $\delta$ Dra, HD 41047, 42 Dra and HD 152222, are promising primary standard candidates. The stars $\theta$ Umi, HD 138265 and HD 159330 prove to be good primary standard candidates, too, but only up to $100 \mu \mathrm{m}$ 
due to significant source confusion at $160 \mu \mathrm{m}$ at the spatial resolution of PACS. For three of the new primary standard candidates essential stellar parameters are known, meaning that a dedicated flux model code could be run. As a good model approximation for nine of our primary standard candidates we can scale the continuum flux models of four PACS fiducial standards with the same or quite similar spectral type. Only for $\delta$ Dra is there no suitable counterpart among the fiducial standard stars. This allows us to establish a set of five FIR primary standard candidates up to $160 \mu \mathrm{m}$, which are 2-20 times fainter than the faintest PACS fiducial standard ( $\gamma$ Dra) with absolute accuracy of $<6 \%$. The accuracy for the faintest primary standard candidate, HD152222 (80 times fainter than $\gamma$ Dra), is currently limited to $13 \%$ by the accuracy of the existing $K$-band photometry. A set of three primary standard candidates up to $100 \mu \mathrm{m}$ with an absolute accuracy of $<7 \%$ complements the list of proven flux standards.

Acknowledgements. PACS has been developed by a consortium of institutes led by MPE (Germany) and including UVIE (Austria); KUL, CSL, IMEC (Belgium); CEA, OAMP (France); MPIA (Germany); IFSI, OAP/AOT, OAA/CAISMI, LENS, SISSA (Italy); IAC (Spain). This development has been supported by the funding agencies BMVIT (Austria), ESA-PRODEX (Belgium), CEA/CNES (France), DLR (Germany), ASI (Italy), and CICYT/MCYT (Spain). ZB acknowledges funding by DLR for this work. TM receives funding from the European Union's Horizon 2020 Research and Innovation Programme, under Grant Agreement no. 687378. This research has made use of the SIMBAD data base and the VizieR catalogue access tool, operated at CDS, Strasbourg, France. This research has made use of SAOImage DS9, developed by Smithsonian Astrophysical Observatory. This research has made use of the NASA/IPAC Infrared Science Archive, which is operated by the Jet Propulsion Laboratory, California Institute of Technology, under contract with the National Aeronautics and Space Administration. We thank the referee for constructive comments.

\section{References}

Alonso, A., Arribas, S., \& Martínez-Roger, C. 1999, A\&AS, 140, 261 Baines, E. K., Döllinger, M. P., Cusano, F., et al. 2010, ApJ, 710, 1365 Balog, Z., Müller, T., Nielbock, M., et al. 2014, Exp. Astron., 37, 129 Beckwith, S. V. W. 1999, The origin of stars and planetary systems, eds. C. J Lada, \& N. D. Kylafis (Dordrecht: Kluwer Academic Publishers), 579

Berta, S., Magnelli, B., Nordon, R., et al. 2011, A\&A, 532, A49

Bessel, M. S. 2005, ARA\&A, 43, 293

Bianchi, S., Giovanardi, C., Smith, M. W. L., et al. 2017, A\&A, 597, A130

Blackwell, D. E., Lynas-Gray, A. E., \& Petford, A. D. 1991, A\&A, 245, 567

Bottom, M., Kuhn, J., Mennesson, B., et al. 2015, ApJ, 809, 11

Bouchet, P., García-Marín, M., Lagage, P.-O., et al. 2015, PASP, 127, 612

Chiang, E. I., \& Goldreich, P. 1997, ApJ, 490, 368

Cohen, M., Walker, R. G., Barlow, M. J., \& Deacon, J. R. 1992, AJ, 104, 1650

Cohen, M., Witteborn, F. C., Walker, R. G., Bregman, J. D., \& Wooden, D. H. 1995, AJ, 110, 275

Cohen, M., Witteborn, F. C., Carbon, D. F., et al. 1996, AJ, 112, 2274

Cohen, M., Walker, R. G., Carter, B., et al. 1999, AJ, 117, 1864

Cohen, M., Carbon, D. F., Welch, W. J., et al. 2005, AJ, 129, 2836

Cutri, R. M., Skrutskie, M. F., van Dyk, S., et al. 2003, VizieR Online Data Catalog: II/246

Decin, L., Vandenbussche, B., Waelkens, C., et al. 2003, A\&A, 400, 709

Dehaes, S., Bauwens, E., Decin, L., et al. 2011, A\&A, 533, A107

Deustua, S., Kent, S., \& Smith, J. A. 2013, Absolute Calibration of Astronomical

Flux Standards, eds. T. D. Oswalt \& H. E. Bond (Dordrecht: Springer), 375

Doi, Y., Takita, S., Ootsubo, T., et al. 2015, PASJ, 67, 50

Döllinger, M. P., Hatzes, A. P., Pasquini, L., et al. 2009, A\&A, 499, 935
Dommanget, J., \& Nys, O. 2002, VizieR Online Data Catalog: I/274

Ducati, J. R. 2002, VizieR Online Data Catalog: II/237

Dyck, H. M., Benson, J. A., Van Belle, G. T., \& Ridgway, S. T. 1996, AJ, 111, 1705

Fruchter, A. S., \& Hook, R. N. 2002, PASP, 114, 144

Gordon, K. D., Engelbracht, C. W., Fadda, D., et al. 2007, PASP, 119, 1019

Graciá-Carpio, J., Wetzstein, M., \& Roussel, H. 2015, ArXiv e-prints [arXiv: 1512.03252]

Gray, R. O., Corbally, C. J., Garrison, R. F., McFadden, M. T., \& Robinson, P. E. 2003, VizieR Online Data Catalog: J/AJ/126/2048

Groenewegen, M. 2012, A\&A, 540, A32

Hammersley, P. L., Jourdain de Muizon, M., Kessler, M. F., et al. 1998, A\&AS, 128,207

Hatzes, A., Cochran, W., Endl, M., et al. 2006, A\&A, 457, 335

Herbstmeier, U., Ábrahám, P., Lemke, D., et al. 1998, A\&A, 332, 739

Herschel-Spot (HSpot) User's Guide: Herschel Observation Planning Tool. 2013, HERSCHEL-HSC-DOC-0788, v 5.2 for HSpot v 7.0, http://herschel. esac. esa.int/Docs/HSPOT/pdf/hspot-help.pdf

Jofré, E., Petrucci, R., Saffe, C., et al. 2015, A\&A, 574, A50

Judge, P. G., Jordan, C., \& Rowan-Robinson, M. 1987, MNRAS, 224, 93

Juvela, M., Mattila, K., Lemke, D., et al. 2009, A\&A, 500, 763

Kiss, C., Klaas, U., \& Lemke, D. 2005, A\&A, 430, 343

Lee, B.-C., Mkritchian, D., Han, A., Kim, K.-M., \& Park, M.-G. 2011, A\&A, 529, A134

Lemke, D., Klaas, U., Abolins, J., et al. 1996, A\&A, 315, L64

Lutz, D. 2015, Herschel Technical Report on PACS Photometer Point Spread Function, PICC-ME-TN-033, http://herschel.esac.esa.int/twiki/ pub/Public/PacsCalibrationWeb/bolopsf_22.pdf

Lyubimkov, L. S., \& Poklad, D. B. 2014, Kinematics Phys. Celestial Bodies, 30 244

Meixner, M., Carter, R., Leisawitz, D., et al. 2017, Am. Astron. Soc. Meet. Abstr., $229,238.20$

Moór, A., Müller, T. G., Kiss, C., et al. 2014, Exp. Astron., 37, 225

Moshir, M., Copan, G., Conrow, T., et al. 1989, VizieR Online Data Catalog: II/156A

Müller, T., Okumura, K., \& Klaas, U. 2011, Herschel Technical Report on PACS Photometer Passbands and Colour Correction Factors for Various Source SEDs, PICC-ME-TN-038.

Müller, T., Balog, Z., Nielbock, M., et al. 2014, Exp. Astron., 37, 253

Müller, T., Balog, Z., Nielbock, M., et al. 2016, A\&A, 588, A109

Neugebauer, G., \& Leighton, R. B. 1969, VizieR Online Data Catalog: II/2B

Nielbock, M., Müller, T., Klaas, U., et al. 2013, Exp. Astron., 36, 631

Ott, S. 2010, Astronomical Data Analysis Software and Systems XIX, eds. Y. Mizumoto, K.-I. Morita, \& M. Ohishi, ASP Conf. Ser., 434, 139

Piazzo, L., Calzoletti, L., Faustini, F., et al. 2015, MNRAS, 447, 1471

Pilbratt, G., Riedinger, J., Passvogel, T., et al. 2010, A\&A, 518, L1

Planck Collaboration XI. 2014, A\&A, 571, A11

Plets, H., Waelkens, C., Oudmaijer, R. D., \& Waters, L. B. F. M. 1997, A\&A, 323,513

Poglitsch, A., Waelkens, C., Geis, N., et al. 2010, A\&A, 518, L2

Popesso, D., Magnelli, B., Buttiglione, S., et al. 2012, ArXiv e-prints [arXiv: 1211.4257]

Roussel, H. 2013, PASP, 125, 1126

Schulz, B., Marton, G., Valtchanov, I., et al. 2017, ArXiv e-prints [arXiv: 1706.00448]

Selby, M. J., Hepburn, I., Blackwell, D. E., et al. 1988, A\&AS, 74, 127

Shirahata, M., Hasegawa, S., Ootsubo, T., et al. 2009, PASJ, 61, 737

Sibthorpe, B., Helmich, F., Roelfsema, P., Kaneda, H., \& Shibai, H. 2015, The SPICA Mission, EAS Pub. Ser., 75, 411

Skrutskie, M. F., Cutri, R. M., Stiening, R., et al. 2006, AJ, 131, 1163

Smirnov, A. V., Baryshev, A. M., Pilipenko, S. V., et al. 2012, in Space Telescopes and Instrumentation 2012: Optical, Infrared, and Millimeter Wave, Proc. SPIE, 8442, 84424C

Takita, S., Doi, Y., Ootsubo, T., et al. 2015, PASJ, 67, 51

Tsuji, T. 1981, A\&A, 99, 48 


\section{Appendix A: Scan map photometry}

\section{A.1. Comparison of different aperture sizes for optimization of photometric aperture}

Table A.1. Comparison of mini scan map photometry for different aperture sizes.

\begin{tabular}{|c|c|c|c|c|c|}
\hline Star & $\begin{array}{r}\text { Filter } \\
(\mu \mathrm{m})\end{array}$ & \# & $\begin{array}{c}\text { phot_1 } \\
(\mathrm{mJy})\end{array}$ & $\begin{array}{c}\text { phot_s } \\
(\mathrm{mJy})\end{array}$ & $\begin{array}{l}\text { Model } \\
(\mathrm{mJy})\end{array}$ \\
\hline \multirow[t]{3}{*}{$\beta \mathrm{Gem}$} & 70 & 8 & $2649.4 \pm 4.1$ & $2648.7 \pm 1.2$ & 2457 \\
\hline & 100 & 8 & $1287.1 \pm 2.4$ & $1283.8 \pm 1.4$ & 1190 \\
\hline & 160 & 16 & $497.4 \pm 5.8$ & $496.9 \pm 2.1$ & 455.9 \\
\hline \multirow[t]{3}{*}{$\alpha$ Ari } & 70 & 8 & $1668.3 \pm 3.7$ & $1663.7 \pm 3.1$ & 1707 \\
\hline & 100 & 8 & $831.6 \pm 4.3$ & $820.2 \pm 1.8$ & 831.4 \\
\hline & 160 & 16 & $336.0 \pm 3.0$ & $328.0 \pm 1.9$ & 321.0 \\
\hline \multirow[t]{3}{*}{$\varepsilon$ Lep } & 70 & 8 & $1157.1 \pm 6.5$ & $1165.6 \pm 2.3$ & 1182 \\
\hline & 100 & 12 & $568.8 \pm 2.7$ & $568.4 \pm 1.6$ & 576.2 \\
\hline & 160 & 18 & $217.5 \pm 6.5$ & $223.5 \pm 2.6$ & 222.7 \\
\hline \multirow[t]{3}{*}{$\omega$ Cap } & 70 & 8 & $839.0 \pm 2.9$ & $845.2 \pm 1.7$ & 857.7 \\
\hline & 100 & 8 & $414.3 \pm 1.8$ & $412.8 \pm 1.4$ & 418.0 \\
\hline & 160 & 16 & $168.6 \pm 4.0$ & $160.5 \pm 1.9$ & 161.5 \\
\hline \multirow[t]{3}{*}{$\eta$ Dra } & 70 & 8 & $517.8 \pm 13.0$ & $506.0 \pm 2.6$ & 479.5 \\
\hline & 100 & 8 & $237.9 \pm 9.1$ & $249.5 \pm 4.5$ & 232.6 \\
\hline & 160 & 16 & $116.4 \pm 7.3$ & $98.4 \pm 5.1$ & 89.4 \\
\hline \multirow[t]{3}{*}{$\delta$ Dra } & 70 & 12 & $433.6 \pm 2.1$ & $436.0 \pm 1.1$ & 428.9 \\
\hline & 100 & 10 & $214.0 \pm 1.6$ & $214.2 \pm 1.4$ & 207.7 \\
\hline & 160 & 22 & $89.0 \pm 5.1$ & $85.4 \pm 2.2$ & 79.6 \\
\hline \multirow[t]{3}{*}{$\theta \mathrm{Umi}$} & 70 & 4 & $278.7 \pm 5.7$ & $284.2 \pm 1.5$ & 286.2 \\
\hline & 100 & 2 & $128.0 \pm 2.4$ & $144.2 \pm 5.0$ & 139.5 \\
\hline & 160 & 2 & $72.8 \pm 10.4$ & $62.3 \pm 2.5$ & 53.9 \\
\hline \multirow[t]{2}{*}{ HD 41047} & 100 & 2 & $99.4 \pm 2.3$ & $96.7 \pm 0.7$ & 95.4 \\
\hline & 160 & 2 & $52.8 \pm 4.1$ & $35.7 \pm 5.6$ & 36.9 \\
\hline \multirow[t]{3}{*}{42 Dra } & 70 & 4 & $146.5 \pm 1.8$ & $147.8 \pm 0.9$ & 153.7 \\
\hline & 100 & 4 & $75.1 \pm 1.7$ & $73.1 \pm 0.9$ & 75.3 \\
\hline & 160 & 8 & $31.2 \pm 1.4$ & $28.2 \pm 1.0$ & 29.4 \\
\hline \multirow[t]{3}{*}{ HD 138265} & 70 & 4 & $109.2 \pm 2.6$ & $112.8 \pm 1.0$ & 115.9 \\
\hline & 100 & 6 & $57.2 \pm 1.6$ & $56.5 \pm 0.5$ & 56.8 \\
\hline & 160 & 5 & $31.2 \pm 6.1$ & $30.8 \pm 1.0$ & 22.2 \\
\hline \multirow[t]{2}{*}{ HD 159330} & 70 & 4 & $60.7 \pm 4.7$ & $64.8 \pm 1.1$ & 64.2 \\
\hline & 100 & 6 & $32.9 \pm 2.3$ & $31.0 \pm 1.1$ & 31.5 \\
\hline \multirow{3}{*}{ HD 152222} & 70 & 4 & $35.1 \pm 1.0$ & $39.0 \pm 0.9$ & 39.4 \\
\hline & 100 & 2 & $22.4 \pm 0.7$ & $20.8 \pm 0.1$ & 19.3 \\
\hline & 160 & 4 & $6.2 \pm 2.2$ & $7.4 \pm 0.8$ & 7.5 \\
\hline \multirow[t]{3}{*}{ HD 39608} & 70 & 3 & $29.8 \pm 3.4$ & $30.5 \pm 1.1$ & 30.9 \\
\hline & 100 & 1 & $18.1 \pm 2.2$ & $17.9 \pm 0.9$ & 15.1 \\
\hline & 160 & 2 & $18.2 \pm 2.6$ & $12.1 \pm 0.7$ & 5.9 \\
\hline HD 181597 & 70 & 2 & $25.9 \pm 3.3$ & $29.1 \pm 2.0$ & 28.0 \\
\hline$\delta$ Hyi & 70 & 1 & $7.7 \pm 2.4$ & $22.2 \pm 1.5$ & 22.9 \\
\hline
\end{tabular}

Notes. phot_l is the photometry with the large aperture sizes $12^{\prime \prime} / 12^{\prime \prime} / 22^{\prime \prime}$, phot_s is the photometry with the small aperture sizes $5.6^{\prime \prime} / 6.8^{\prime \prime} / 10.7^{\prime \prime}$. Stellar fluxes $f_{\text {star }}$ are determined as the colourcorrected weighted average of aperture corrected fluxes $\mathrm{f}_{\text {tot }}$ from \# of individual scan maps. Model fluxes are from Table 1. Quoted uncertainties are the weighted standard deviations for $\# \geq 2$ and $\sigma_{\text {aper,corr }}$ (Eq. (4)) for \#=1.

\section{A.2. Dependence on applied mapper software}

For the faintest star at $160 \mu \mathrm{m}$ with reliable photometry in all three filters, HD 152222, we conduct an inter-comparison of the HPF photometry from the deepest map with the results of three additional Herschel mapper softwares, namely
Table A.2. Comparison of the photometric results of HD 152222 from different mapper softwares: High Pass Filter (HPF, default reduction scheme of this work), JScanam (Graciá-Carpio et al. 2015), Scanamorphos (Roussel 2013) and Unimap (Piazzo et al. 2015).

\begin{tabular}{rrrccc}
\hline \hline Mapper Filter & $r_{\text {aper }}$ & $\begin{array}{c}f_{\text {star }} \\
(\mu \mathrm{m})\end{array}$ & $\begin{array}{c}\sigma_{\text {aper }} \\
\left({ }^{\prime \prime}\right)\end{array}$ & $\begin{array}{c}\frac{f_{\text {star }}^{\text {maper }}}{f_{\text {star }}^{H}} \\
(\mathrm{mJJy})\end{array}$ \\
\hline HPF & 70 & 5.6 & 37.7 & \pm 0.21 & - \\
& 100 & 6.8 & 20.7 & \pm 0.15 & - \\
JScanam & 160 & 10.7 & 6.5 & \pm 0.27 & - \\
& 70 & 5.6 & 39.3 & \pm 0.24 & $1.04 \pm 0.02$ \\
& 100 & 6.8 & 20.5 & \pm 0.16 & $0.99 \pm 0.02$ \\
Scanamorphos & 160 & 10.7 & 6.5 & \pm 0.31 & $1.00 \pm 0.14$ \\
& 70 & 5.6 & 35.5 & \pm 0.19 & $0.94 \pm 0.02$ \\
& 100 & 6.8 & 17.6 & \pm 0.13 & $0.85 \pm 0.02$ \\
Unimap & 160 & 10.7 & 4.2 & \pm 0.28 & $0.65 \pm 0.11$ \\
& 70 & 5.6 & 39.9 & \pm 0.45 & $1.06 \pm 0.03$ \\
& 100 & 6.8 & 20.5 & \pm 0.23 & $0.99 \pm 0.03$ \\
& 160 & 10.7 & 7.5 & \pm 0.49 & $1.15 \pm 0.19$ \\
\hline
\end{tabular}

Notes. Used OBSIDs are the combinations of $1342240702+03$ at $70 \mu \mathrm{m}$ and $1342227973+74$ at 100 and $160 \mu \mathrm{m}$. Listed fluxes are the colourcorrected total stellar fluxes $f_{\text {star }}$.

JScanam (Graciá-Carpio et al. 2015), Scanamorphos ${ }^{11}$ (Roussel 2013) and Unimap ${ }^{11}$ (Piazzo et al. 2015). The data analysis was done by applying the standard HIPE ipipe (interactive pipeline) scripts of these mappers ${ }^{12}$ and selecting the same output pixel sizes as defined in Table 2.

For the Scanamorphos processing release version 25 of the software was applied, the "mini-map" option was selected, and the software was set to correct for the PACS distortion flat-field. For the JScanam processing version 14.2.0 (analogue to HIPE version) was applied and the "galactic" option was switched on. For the Unimap processing, version 6.5.3 was applied with the parameter pixelNoise (gain to apply to the estimated pixel noise in the GLS pixel noise compensation ${ }^{12}$ ) set to zero. For the comparison with the other mappers we used the weighted GLS (Generalized Least-Squares) L2.5 map product, corresponding to the FITS XTENSION "Image".

Since our analysis is restricted to one map in each filter, we do not intend to give a full quantitative performance assessment of the four mapper softwares, but rather point out some qualitative findings for these faint star map products.

Fig. A.1 shows the inter-comparison of the maps produced by the default HPF mapper software and the three other mapper softwares for the three PACS filters. The star can be clearly identified as the central source in all three filters for all four mappers.

Table A.2 lists the colour-corrected total stellar fluxes $f_{\text {star }}$ derived from aperture photometry with 5.'6, 6.'8 and 10.'7 aperture radius at 70,100 and $160 \mu \mathrm{m}$, respectively.

We also list the noise inside the measurement aperture, $\sigma_{\text {aper }}$ (cf. Eq. (2)), which was determined from all maps with the histogram method described in Sect. 3.3. We note that here only

\footnotetext{
${ }_{11}$ Herschel user contributed software https://www . cosmos. esa.int/web/herschel/ user-contributed-software

${ }^{12}$ Herschel data processing overview

https://www . cosmos. esa. int/web/herschel/dataprocessing-overview,

in particular PACS Data Reduction Guide Photometry.
} 

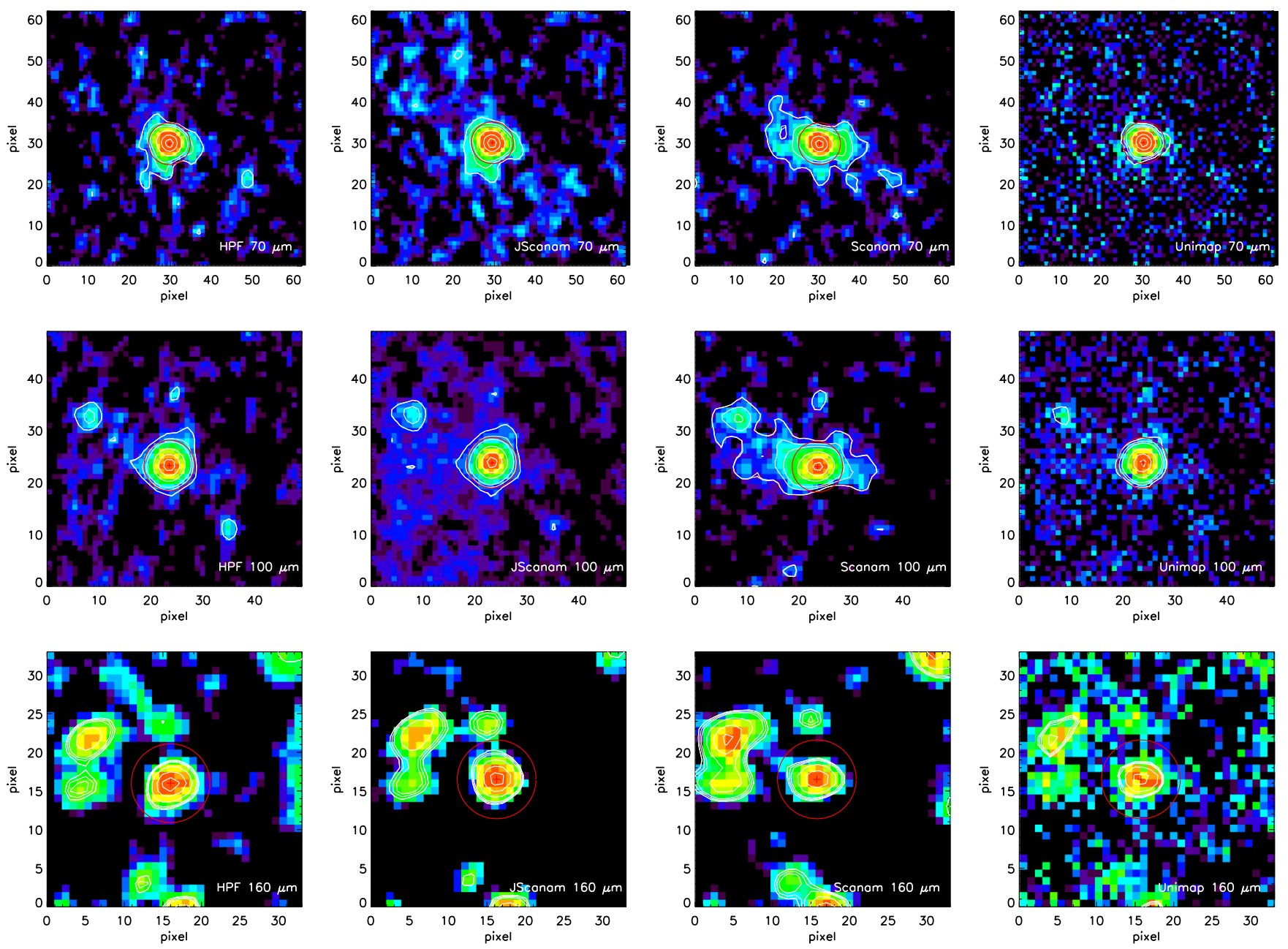

Fig. A.1. Inter-comparison of HD 152222 photometric maps for different mapper softwares for 70, 100, and $160 \mu \mathrm{m}$ (top to bottom). First column: High Pass Filter (HPF, default reduction scheme of this work), second column: JScanam, third column: Scanamorphos and 4th column: Unimap. Used OBSIDs are the combinations of $1342240702+03$ at $70 \mu \mathrm{m}$ and $1342227973+74$ at 100 and $160 \mu \mathrm{m}$. The red circle indicates the photometric aperture.

this noise term can be used for inter-comparison, not the one corrected for correlated noise, $\sigma_{\text {aper,corr }}$, since correlated noise correction factors $f_{\text {corr }}$ were only derived for the high-pass filtered data reduction (one may argue that the final corrected noise $\sigma_{\text {aper,corr }}$ should be about the same for all mappers, since it is mainly determined by the detector noise). A $\sigma_{\text {tot }}$ for the total flux can be calculated as $\sigma_{\text {tot }}=c_{\text {aper }} \times \sigma_{\text {aper }}$.

The noise determined from the JScanam maps is slightly larger than that of the HPF maps. This finding indicates that the noise correlation is slightly less for the JScanam mapper. The noise determined from the Scanamorphos maps is slightly smaller than that of the HPF maps, indicating a slightly higher noise correlation. The noise determined from the Unimap maps is a factor of 1.5-2.1 higher than that of the HPF maps. This is explained by Unimap using the Generalized Least-Squares (GLS) algorithm to remove the correlated $\frac{1}{f}$-noise (Piazzo et al. 2015). The Unimap noise is hence closer to the real noise level, and the above scaling factors do not exceed the correlated noise correction factors $f_{\text {corr }}$ to be applied to the HPF noise (cf. Eq. (4) and Table 2) for calculation of the correlation-free noise level.

At $70 \mu \mathrm{m}$, with an expected source flux in the order of $40 \mathrm{mJy}$, the fluxes of all four mappers correspond to each other within $4-6 \%$. At $100 \mu \mathrm{m}$, with an expected source flux in the order of $20 \mathrm{mJy}$, the correspondence is still better than $15 \%$.
At $160 \mu \mathrm{m}$, with an expected source flux of only $\approx 8 \mathrm{mJy}$ and $S / N \lesssim 10$, the scatter is naturally larger. Jscanam photometry shows the best correspondence with the HPF photometry, being within $4 \%$ for all filters. This can be expected, since both mappers use the same projection algorithm photProject(). Unimap photometry shows the second best correspondence with HPF photometry, with the tendency that the Unimap fluxes are larger (at 70 and $160 \mu \mathrm{m}$ ). Scanamorphos photometry gives systematically smaller fluxes than HPF photometry, with the deviation increasing with wavelength and a $160 \mu \mathrm{m}$ flux which is noticeably off.

The PACS photometric calibration scheme (Balog et al. 2014) was established with HPF analysis, in particular also the derivation of the aperture photometry correction factors $c_{\text {aper }}$ from the PACS Point Spread Function (Lutz 2015), by determining the Encircled Energy Fractions with radius. Therefore, one aspect affecting the aperture photometry depending on the selected mapper software was not considered in the evaluation scheme described above, namely the shape of the point spread function. From the inspection of the stellar intensity profiles and their close surrounding in Fig. A.1, in particular from the 70 and $100 \mu \mathrm{m}$ images, it is obvious that there are systematic differences in the resulting profile shapes of the star depending on the applied mapper. The HPF processing shows the typical tri-lobe 
pattern of the PACS point spread function (Lutz 2015). The JScanam processing shows the closest appearance. The Unimap profiles look slightly sharper and show less pronounced lobes. The Scanamorphos profiles on the other hand appear somewhat less concentrated than the HPF profiles and with more pronounced lobes.

This means that for the other mappers adapted aperture correction factors should be applied, which alters the photometric results somewhat. The ratios $\frac{f_{\text {totcc }}^{\text {mapper }}}{f_{\text {tot, cc }}^{H P F}}$ in Table A.2 should not be used as general scaling factors between the various mappers, since they are based on the evaluation of a single map of a very faint star implying quite some uncertainty. Accurate scaling factors for photometry with the various mappers do not exist yet and will be determined on the basis of the high signal-to-noise fiducial standard observational database in a forthcoming paper (Balog et al., 2018, in prep.).

\section{A.3. Photometry results of individual measurements}

Individual photometric results for the 70,100 , and $160 \mu \mathrm{m}$ filters are compiled in Tables A.3 to A.5 which are available in electronic form at CDS. The applied radius for the photometric aperture was 5.6, 6.8 and 10.7 for the 70,100 , and $160 \mu \mathrm{m}$ filter, respectively. The number of output pixels (1."1, 1'. 4, and 2 '. 1 size, respectively) inside this photometric aperture is $\mathrm{N}_{\text {aper }}=$ $81.42,74.12$, and 81.56, respectively. The corresponding correction factors for correlated noise are $f_{\text {corr }}=3.13,2.76$, and 4.12 , respectively. Aperture correction factors are $c_{\text {aper }}=1.61,1.56$, and 1.56 for the 70, 100, and $160 \mu \mathrm{m}$ filter, respectively. Proper motion correction was applied throughout.

The tables contain the following information: Col. 1: Unique observational identifier (OBSID) of the PACS observation; Col. 2: Herschel Observational Day (OD); Col. 3: Target name; Col. 4: Applied gain $(\mathrm{G})$ of the PACS bolometer electronics: $\mathrm{h}($ igh $) / \mathrm{l}(\mathrm{ow})$; Col. 5: Scan speed: low $=10 " 1 / \mathrm{s}$, medium $=20 " 1 \mathrm{~s}$, high $=60 \mathrm{\prime \prime} / \mathrm{s}$; Col. 6: Number of repetitions (rep.) of the basic scan map with the parameters given in next column; Col. 7: Parameters of the scan map: scan leg length(") / scan leg separation (") / number of scan legs; Col. 8: Scan angle of the map, in case of co-added maps all angles of the individual maps are given; Col. 9: Measured flux inside the photometric aperture of this filter, $f_{\text {aper }}$; Col. 10: Noise per pixel, $\sigma_{\text {pix }}$; Col. 11: Noise corrected for correlated noise inside the measurement aperture, $\sigma_{\text {aper,corr }}$, according to Eq. (4). Col. 12: Achieved signal-to-noise ratio according to Eq. (5); Col. 13: Stellar flux $\mathrm{f}_{\text {star }}$ according to Eq. (1); Cols. 14-16: Maximum and minimum full width (W) half maximum (in ") of the source PSF and its uncertainty determined by an elliptical fit to the intensity profile.

\section{Appendix B: Chopped photometry}

\section{B.1. Photometry results of individual measurements}

Individual photometric results for the 70,100 , and $160 \mu \mathrm{m}$ filters are compiled in Tables B.1 to B.3 which are available in electronic form at CDS. The applied radius for the photometric aperture was 5.6, 6.8, and $10^{\prime} .7$ for the 70,100 , and $160 \mu \mathrm{m}$ filter, respectively. The number of output pixels (1'. $1,1^{\prime \prime} .4$, and 2 '. 1 size, respectively) inside this photometric aperture is $N_{\text {aper }}=$ $81.42,74.12$, and 81.56, respectively. The corresponding correction factor for correlated noise are $f_{\text {corr }}=6.33,4.22$, and 7.81, respectively. Aperture correction factors are $c_{\text {aper }}=1.61,1.56$ and 1.56 for the 70,100 , and $160 \mu \mathrm{m}$ filter, respectively. Proper motion correction was applied throughout.

The tables contain the following information: Col. 1: Unique observational identifier (OBSID) of the PACS observation; Col. 2: Herschel Observational Day (OD), including its phase; Col. 3: Target name; Col. 4: Applied gain (G) of the PACS bolometer electronics: h(igh)/l(ow); Col. 5: Chopper dither pattern: $y($ es $) / n(o)$; Col. 6: Number of repetitions (rep.) of the basic chop/nod cycle; Col. 7: Fitted peak flux intensity of the source; Col. 8: Measured flux inside the photometric aperture of this filter, $f_{\text {aper }}$; Col. 9: Noise per pixel, $\sigma_{\text {pix }}$; Col. 10: Noise corrected for correlated noise inside the measurement aperture, $f_{\text {aper }}$, according to Eq.( 4). Col. 11: Achieved signal-to-noise ratio according to Eq.( 5); Col. 12: Stellar flux $f_{\text {star }}$ according to Eq. ( 1); Cols. $13+14$ : Maximum and minimum full width (W) half maximum (in ") of the source PSF.

\section{Appendix C: Comparison scan map with chop/nod photometry}

In Table C.1 we list the flux ratios of scan map photometry and chop/nod photometry for ten sources, which were observed in both modes. The comparison between the two photometry modes gives the following result:

For $70 \mu \mathrm{m}$ photometry, the consistency of the fluxes is better than $3 \%$ for seven out of nine sources. The two excursions, HD 159330 and HD 15008, are consistent within the larger error margin which is caused by a larger uncertainty because of only one chop/nod measurement (HD 159330) or the faintness of the source (HD 15008).

For $100 \mu \mathrm{m}$ photometry, the consistency of the fluxes is better than $2 \%$ for five out of eight sources. For HD 138265 the flux consistency is $\approx 4 \%$, but the derived error margin is smaller. For $\eta$ Dra and HD 41047 there is only one chop/nod measurement, which introduces a high uncertainty, but fluxes are consistent within the error margin.

For $160 \mu \mathrm{m}$ photometry, the consistency of the fluxes is better than $3 \%$ for four out of seven sources. For $\varepsilon$ Lep the scan map flux is $9 \%$ higher than the chop/nod one. There is only a small (4) number of chop/nod measurements vs a large (18) number of scan map measurements. We therefore consider the scan map mode result as the more reliable one. The opposite is the case for the number of photometric measurements of $\theta \mathrm{Umi}$, with 2 scan map measurements vs 16 chop/nod measurements.

Table C.1. Ratios of fluxes obtained in scan map mode photometry (Table 7) vs chop/nod mode photometry (Table 8) as a measure of consistency between the two photometry modes.

\begin{tabular}{rcccc}
\hline \hline HD & Name & $R_{70}^{S / C}$ & $R_{100}^{S / C}$ & $R_{160}^{S / C}$ \\
\hline 62509 & $\beta$ Gem & $1.031 \pm 0.005$ & $1.014 \pm 0.011$ & $1.000 \pm 0.006$ \\
32887 & $\varepsilon$ Lep & $0.987 \pm 0.006$ & $1.019 \pm 0.025$ & $1.093 \pm 0.038$ \\
148387 & $\eta$ Dra & $0.993 \pm 0.034$ & $1.059 \pm 0.068$ & $0.969 \pm 0.095$ \\
180711 & $\delta$ Dra & $0.992 \pm 0.007$ & $1.002 \pm 0.009$ & $1.026 \pm 0.043$ \\
139669 & $\theta$ Umi & $1.009 \pm 0.008$ & $1.018 \pm 0.043$ & $1.131 \pm 0.070$ \\
41047 & HR 2131 & - & $1.101 \pm 0.063$ & $1.248 \pm 0.652$ \\
138265 & HR 5755 & $1.001 \pm 0.011$ & $0.958 \pm 0.011$ & $1.000 \pm 0.050$ \\
159330 & HR 6540 & $1.076 \pm 0.118$ & $1.016 \pm 0.116$ & - \\
152222 & - & $1.008 \pm 0.099$ & - & - \\
15008 & $\delta$ Hyi & $1.110 \pm 0.064$ & - & -
\end{tabular}


Here the scan map flux is $13 \%$ higher than the chop/nod one. However, the 2 scan map measurements, each with 6 repetitions, have the best $\mathrm{S} / \mathrm{N}$ of all measurements and are therefore quite reliable. Fifteen out of 16 chop/nod measurements have a repetition factor of only 1 . They still allow a reasonable detection of the source at the expected location but show considerable scatter in the resulting (colour-corrected) fluxes between 35.5 and $91.6 \mathrm{mJy}$ (expected flux according to the model: $53.9 \mathrm{mJy}$ ). Only one chop/nod measurement has 20 repetitions with a S/N comparable to the two scan maps. Its resulting flux of $52.8 \mathrm{mJy}$ is $13 \%$ lower than the average $60.9 \mathrm{mJy}$ from the two scan maps. Here we should note that the annulus used for background determination is closer to the source and narrower for chop/nod aperture photometry (radius 24-28", Nielbock et al. 2013) than for scan map photometry (radius 35-45", Balog et al. 2014). As we discuss in Sect. 5.2, the scan map measurements prove contamination of the source flux by FIR cirrus emission in the order of $10 \%$ explaining the excess over the model flux. The maps also show that there is additional emission around the source which is much more picked up by the background annulus of the chop/nod photometry, resulting in a higher subtracted background value. This leads to the result that the chop/nod photometry is close to the expected model flux, because the underlying cirrus emission is by chance properly compensated for by the background subtraction, while the scan map photometry reveals the extra emission inside the aperture. The photometric result must therefore be associated by an additional uncertainty of $10 \%$, because the background subtraction strongly depends on the selected background area geometry (cf. Table 7). For HD 41047 there is only one chop/nod measurement with a very high assigned flux uncertainty, so that also the flux ratio of scan map to chop/nod photometry is highly uncertain.

\section{Appendix D: ISOPHOT Highly Processed Data Product (HPDP) photometry}

Table D.1. ISOPHOT (Lemke et al. 1996) Highly Processed Data Product (HPDP) photometry of P22 mini-maps of normal stars (https://www.cosmos.esa.int/web/iso/ highly-processed-data-products: Moór et al., 2003, "Farinfrared observations of normal stars measured with ISOPHOT in mini-map mode").

\begin{tabular}{llcccc}
\hline \hline Star & Filter & $\begin{array}{c}\lambda_{\mathrm{c}} \\
(\mu \mathrm{m})\end{array}$ & ISO TDT no. & $\begin{array}{c}f_{v} \\
(\mathrm{mJy})\end{array}$ & $\mathrm{cc}$ \\
\hline$\alpha$ Ari & C_180 & 180 & 79001902 & $314 \pm 19$ & 1.10 \\
$\varepsilon$ Lep & C_60 & 60 & 65701315 & $1779 \pm 71$ & 1.06 \\
& C_50 & 65 & 65701312 & $1835 \pm 65$ & 1.29 \\
& C_70 & 80 & 65701309 & $1113 \pm 46$ & 1.23 \\
& C_90 & 90 & 65701318 & $918 \pm 64$ & 1.17 \\
& C_100 & 100 & 65701306 & $692 \pm 36$ & 1.10 \\
& C_105 & 105 & 65701303 & $601 \pm 44$ & 1.05 \\
& C_120 & 120 & 65002709 & $507 \pm 43$ & 1.21 \\
& C_135 & 150 & 65002103 & $292 \pm 21$ & 1.10 \\
& C_160 & 170 & 65002406 & $225 \pm 21$ & 1.20 \\
$\omega$ Cap & C_60 & 60 & 72701415 & $1232 \pm 65$ & 1.06 \\
& C_50 & 65 & 72701412 & $1258 \pm 45$ & 1.29 \\
& C_70 & 80 & 72701409 & $779 \pm 32$ & 1.23 \\
& C_90 & 90 & 72701418 & $543 \pm 38$ & 1.17 \\
& C_100 & 100 & 72701406 & $490 \pm 25$ & 1.10 \\
& C_105 & 105 & 72701403 & $441 \pm 32$ & 1.05 \\
& C_120 & 120 & 73401709 & $337 \pm 28$ & 1.21 \\
& C_135 & 150 & 73401603 & $221 \pm 16$ & 1.10 \\
& C_160 & 170 & 73401706 & $219 \pm 26$ & 1.20 \\
$\eta$ Dra & C_90 & 90 & 78300677 & $365 \pm 26$ & 1.17 \\
& C_160 & 170 & 35800501 & $123 \pm 16$ & 1.20 \\
\hline
\end{tabular}

Notes. The values in column $f_{v}$ are the original HPDP fluxes (for SED $\left.\propto v^{-1}\right)$. They have to be divided by the colour-correction factor cc, which is for a $5000 \mathrm{~K}$ BB SED. ${ }^{(1)}$ Measurement not used in Fig. 11. 